\title{
Predictions for anisotropic X-ray signatures in the circumgalactic medium: imprints of supermassive black hole driven outflows
}

\author{
Nhut Truong $^{1 \star}$, Annalisa Pillepich ${ }^{1}$, Dylan Nelson ${ }^{2}$, Norbert Werner $^{3}$, and Lars Hernquist ${ }^{4}$ \\ ${ }^{1}$ Max-Planck-Institut für Astronomie, Königstuhl 17, 69117 Heidelberg, Germany \\ ${ }^{2}$ Universität Heidelberg, Zentrum für Astronomie, Institut für theoretische Astrophysik, Albert-Ueberle-Str. 2, 69120 Heidelberg, Germany \\ ${ }^{3}$ Department of Theoretical Physics and Astrophysics, Faculty of Science, Masaryk University, Kotlářská 2, Brno, 611 37, Czech Republic \\ ${ }^{4}$ Institute for Theory and Computation, Harvard-Smithsonian Center for Astrophysics, 60 Garden Street, Cambridge, MA 02138, USA
}

\begin{abstract}
The circumgalactic medium (CGM) encodes signatures of the galaxy-formation process, including the interaction of galactic outflows driven by stellar and supermassive black hole (SMBH) feedback with the gaseous halo. Moving beyond spherically symmetric radial profiles, we study the angular dependence of CGM properties around $z=0$ massive galaxies in the IllustrisTNG simulations. We characterize the angular signal of density, temperature, and metallicity of the CGM as a function of galaxy stellar mass, halo mass, distance, and SMBH mass, via stacking. TNG predicts that the CGM is anisotropic in its thermodynamical properties and chemical content over a large mass range, $M_{*} \sim 10^{10-11.5} M_{\odot}$. Along the minor axis directions, gas density is diluted, whereas temperature and metallicity are enhanced. These feedback-induced anisotropies in the CGM have a magnitude of $0.1-0.3$ dex, extend out to the halo virial radius, and peak at Milky Way-like masses, $M_{*} \sim 10^{10.8} M_{\odot}$. In TNG, this mass scale corresponds to the onset of efficient SMBH feedback and the production of strong outflows. By comparing the anisotropic signals predicted by TNG versus other simulations - Illustris and EAGLE - we find that each simulation produces distinct signatures and mass dependencies, implying that this phenomenon is sensitive to the underlying physical models. Finally, we explore X-ray emission as an observable of this CGM anistropy, finding that future X-ray observations, including the eROSITA all-sky survey, will be able to detect and characterize this signal, particularly in terms of an angular modulation of the X-ray hardness.
\end{abstract}

Key words: galaxies: evolution - galaxies: formation - galaxies: haloes - galaxies: circumgalactic medium-galaxies: supermassive black holes - X-ray: galaxies — methods: numerical

\section{INTRODUCTION}

The circumgalactic medium (CGM) is the gaseous atmosphere that extends far beyond the stellar body of a galaxy, up to hundreds of kiloparsecs in galactocentric distance, and plays an essential role in the process of galaxy evolution (see Tumlinson et al. 2017 for a review). The CGM is a unique laboratory where the cosmic baryon cycle of gas inflows and gas outflows plays out. While gas accretion provides the fuel for future star formation and the overall growth of galaxies, gaseous outflows act in opposition, removing baryons from the dense star-forming interstellar medium. Galactic-scale outflows, also referred to as galactic winds, are sourced primarily by stellar feedback in low-mass galaxies (Zhang 2018), active galactic nuclei (AGN) feedback in more massive systems (Morganti 2017), or a combination of the two at intermediate masses.

AGN feedback, powered by gas accretion onto supermassive black holes (SMBHs), is an important ingredient in current theoretical models of galaxy formation and evolution (Springel 2005; Springel et al. 2005; Booth \& Schaye 2009; Sijacki et al. 2015; Weinberger et al. 2017; Davé et al. 2019). It enables cosmological simulations to reproduce various observed galaxy properties, both of the stellar body as well as their gaseous atmospheres. It is thought to be the physical mechanism that quenches star formation in massive galaxies (Silk \& Rees 1998; Di Matteo et al. 2005; Man \& Belli 2018).

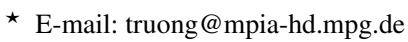

Without AGN feedback, simulations produce overly-massive galaxies inconsistent with observations (Khalatyan et al. 2008; Le Brun et al. 2014; Pillepich et al. 2018a). In addition, AGN feedback is necessary for simulations to reproduce the observed thermodynamical properties of halo gas, from galaxies to groups and cluster mass scales (McCarthy et al. 2010; Le Brun et al. 2014; Henden et al. 2018; Nelson et al. 2018b; Truong et al. 2018, 2020).

Observations have inferred that most, if not all, massive galaxies contain a SMBH at their centers (Kormendy \& Ho 2013; van den Bosch 2016). Nonetheless, both observationally and theoretically, the exact nature of how AGN feedback interacts with the galaxy, much less the surrounding CGM, remains unclear.

Observationally, it is suggested that AGN feedback appears in two modes: "quasar" and "radio" (Croton et al. 2006; Cattaneo et al. 2009; Fabian 2012; McNamara \& Nulsen 2012; Harrison 2017; Morganti 2017). The quasar (or radiative) mode is mostly found in galaxies hosting luminous AGN, where the SMBH has a high accretion rate $\gtrsim 1-10 \%$ of Eddington. In this case, feedback energy is mainly released in the form of radiation from the accretion disk. In contrast, a radio mode may instead operate for SMBHs with low accretion rates. In this state, observations suggest that feedback is released in the form of mechanical energy by means of bubbles, cavities, or relativistic jets, with large amounts of energy $\left(\sim 10^{60} \mathrm{erg} / \mathrm{s}\right)$ deposited into the surrounding environments via e.g. turbulence and/or shocks (Nulsen et al. 2009; Randall et al. 2011; Zhuravleva et al. 2014; Hlavacek-Larrondo et al. 2015).

From the perspective of galaxy evolution simulations, successful 
models for SMBH feedback and galaxy quenching are non-trivial. First, finite numerical resolution implies that this multi-scale feedback physics must be captured in large part by sub-resolution models (e.g., Schaye et al. 2015). Different implementations can differ in a number of ways: the number of feedback channels or modes, the form of feedback energy (e.g. thermal versus kinetic or mechanical), the time variability (e.g. continuous or bursty), and the directionality (e.g. isotropic versus bipolar). The diversity of SMBH feedback models among current cosmological simulations, as well as the diversity of their outcomes, makes it clear that the underlying physics and any subsequent regulation of galaxy growth are still uncertain (Naab \& Ostriker 2017).

The CGM, also called the intracluster medium or ICM for higher galaxy or halo masses, provides an ideal laboratory to study the impact and consequences of SMBH feedback. Some fraction of the feedback energy and momentum injected by a galaxy is expected to be absorbed by this gaseous halo, and galactic winds (or outflows) can modify the global thermodynamical properties of the CGM (Werner et al. 2019; Eckert et al. 2021). In addition, outflows also enrich the gaseous halo by transporting heavy elements synthesised within the star-forming regions. Early X-ray observations provided strong evidence for SMBH-powered ultra-fast outflows (e.g., Reeves et al. 2009; Tombesi et al. 2011, 2012). They also showed that the distribution of heavy elements in the cores of galaxy clusters can be spatially variable, often aligned with radio jets and cavity axes (Simionescu et al. 2008, 2009; Kirkpatrick et al. 2009; O'Sullivan et al. 2011; Kirkpatrick et al. 2011).

In this paper, we use the IllustrisTNG cosmological hydrodynamical simulations (https://www.tng-project.org/) to quantify the spatially-anisotropic distribution of CGM properties. We devote special attention to massive galaxies, where SMBH feedback is the predominant mechanism that drives galactic outflows (Nelson et al. 2019b; Pillepich et al. 2021). In practice, we study if and how the properties of the halo gas depend on its angular position with respect to the central galaxy; i.e. its stellar minor axis. We characterize the CGM structure across the galaxy population by stacking large samples of simulated galaxies, and examine how anisotropies in the CGM can differentiate among different SMBH feedback models, by comparing IllustrisTNG predictions with two other publicly available cosmological simulations: Illustris and EAGLE. Finally, we identify observables with which the predicted CGM anisotropy can be tested.

Our focus on the IllustrisTNG simulation model is motivated by the fact that this model has been shown to contain many baryonic signatures in the gaseous halos of galaxies, while at the same time broadly reproducing many observed features of these atmospheres. The latter include, for example, the abundances of highly ionized oxygen in OVI, OVII, and OVIII (Nelson et al. 2018b), the X-ray properties of hot halos (Truong et al. 2020, 2021), the cool-core fractions of clusters at intermediate redshifts (Barnes et al. 2018), the abundance of cool MgII-traced gas around luminous red galaxies (Nelson et al. 2020) and MgII emission from the CGM (Nelson et al. 2021b), and the high redshift Lyman- $\alpha$ halos in emission (Byrohl et al. 2020). Crucially, according to the IllustrisTNG models, galactic outflows (Nelson et al. 2019b) and the CGM metallicity of low-mass galaxies (Péroux et al. 2020) are anisotropic, with larger mass outflow rates and higher gas metallicities along the minor axis of galaxies. Furthermore, IllustrisTNG predicts the presence of galactic centerlike bubbles in Milky Way/M31-mass galaxies (Pillepich et al. 2021) and that SMBH feedback carves lower-density regions in the CGM around massive galaxies, particularly along their minor axis (Nelson et al. 2019b; Martín-Navarro et al. 2021).

The paper is arranged as follows. We first introduce the simula- tions and our analysis methods in Section 2. In Section 3.1 we present our main result on anistropic CGM properties: density, temperature, and metallicity. We then study how this signal varies as a function of galaxy properties (Section 3.2), its connection to SMBH activity (Section 3.3), and related signatures in other gas properties: pressure and entropy (Section 3.4). We compare the three different cosmological simulations in Section 4, and conclude by discussing X-ray emission predictions in Section 5. Our main results are summarized in Section 6.

\section{METHODS}

\subsection{The TNG100 simulation of IllustrisTNG}

We primarily use simulated data from TNG100, a large-volume cosmological magneto-hydrodynamical (MHD) simulation with an extent of $\sim 110.7 \mathrm{cMpc}$ per side. TNG100 is one of the three primary simulations of the IllustrisTNG project (hereafter TNG, Nelson et al. 2018a; Springel et al. 2018; Marinacci et al. 2018; Naiman et al. 2018; Pillepich et al. 2018b). The simulations are run with AREPo code (Springel 2010), in which dark matter, baryons (gas and stars), and black holes are evolved self-consistently according to the equations of self-gravity and MHD within a $\Lambda$ CDM universe with cosmological parameters consistent with Planck constraints (Planck Collaboration et al. 2016). All three TNG simulations are publicly available (Nelson et al. 2019a).

The volume covered by TNG100 is compatible with current X-ray observations of nearby galaxies (within $100 \mathrm{Mpc}$, see Truong et al. 2020 for a comparison between TNG100 and current X-ray observations) and also well within the grasp of future all-sky survey of eROSITA X-ray telescope (Oppenheimer et al. 2020). Furthermore, the relatively good resolution of TNG100 (baryon mass resolution $m_{\text {baryon }}=1.4 \times 10^{6} M_{\odot}$ ) allows us to comfortably study CGM gas properties in galaxies down to stellar mass $M_{*} \sim 10^{10} M_{\odot}$.

The TNG model for galaxy formation physics (Weinberger et al. 2017; Pillepich et al. 2018a) implements wide-ranging astrophysical processes that are particularly relevant to studies of CGM properties: radiative microphysics including primordial and metal-line radiative cooling in the presence of ionizationing UV background radiation field; star formation and stellar evolution, including chemical enrichment and supernovae feedback (Pillepich et al. 2018a); the formation and growth of SMBHs as well as their associated feedback. We briefly present the key features of the stellar and SMBH feedback in the following paragraphs.

The TNG model of stellar feedback is based on the original Illustris model (Vogelsberger et al. 2013) with a few modifications (see Pillepich et al. 2018a for a detailed description). Stellar feedback is realized via wind particles, which are launched isotropically from the star-forming gas cells. The feedback energy carried by the wind is proportional to the local, instantaneous star-forming rate and contains a kinetic as well as thermal component.

In TNG, SMBHs are seeded with a mass of $M_{\mathrm{BH} \text {,seed }} \sim 10^{6} \mathrm{M}_{\odot}$ in halos that exceed a total mass of $7 \times 10^{10} \mathrm{M}_{\odot}$. SMBHs then grow via gas accretion governed by a Eddington-limited Bondi model (see the governing Equations 1-3 in Weinberger et al. 2018), and/or via merging with other SMBHs, following the mergers of their host galaxies and haloes. The TNG model adopts a dual-mode SMBH feedback mechanism, injecting thermal and kinetic energy at high and low accretion rates, respectively (Weinberger et al. 2017). In both modes the amount of feedback enery is proportional to the accretion rate (as described by Equations 7-9 in Weinberger et al. 2017). SMBHs 
transition to the low-state kinetic mode when their Eddington ratio falls below a threshold of $\chi=\min \left[0.002\left(M_{\mathrm{BH}} / 10^{8} M_{\odot}\right)^{2}, 0.1\right]$. Due to the black hole galaxy stellar mass relation, this occurs at a characteristic mass scale $M_{\star} \sim 10^{10.5} \mathrm{M}_{\odot}$ corresponding to hosted SMBHs with $M_{\mathrm{BH}} \sim 10^{8} \mathrm{M}_{\odot}$ (Nelson et al. 2018a; Weinberger et al. 2018). This transition results in the quenching of galaxies, halting their star-formation while also shaping the thermodynamical properties of their gaseous halos (Nelson et al. 2018b; Zinger et al. 2020; Truong et al. 2020; Nelson et al. 2021a).

\subsection{Galaxy selection and gas properties}

In this work we consider only central galaxies at $z=0$ and with halo mass $M_{200 c} \gtrsim 10^{11.5} M_{\odot}$, where $M_{200 c}$ is the total mass within a spherical region with mean density equal to 200 times the critical density of the Universe $\left(\rho_{\text {crit }}\right)$ at the considered redshift: $M_{200 \mathrm{c}}=$ $4 / 3 \pi R_{200 \mathrm{c}}^{3} 200 \rho_{\text {crit }}(z)$ where $R_{200 c}$ is the radius of the sphere. At $z=0$, this halo mass selection corresponds to stellar mass $M_{*} \gtrsim$ $10^{10} M_{\odot}$, where we define $M_{*}$ as the mass of stars within a spherical aperture of physical radius $30 \mathrm{kpc}$. We also define the following relevant galaxy properties:

- Star formation status. The star formation status of a galaxy is defined according to its relative distance, based on the instantaneous star formation rate (SFR) versus stellar mass plane, from the starforming main sequence. Following Pillepich et al. (2019) we adopt:

- Star-forming: $\Delta \log _{10}$ SFR $>-0.5$.

- Quenched: $\Delta \log _{10}$ SFR $\leqslant-1.0$.

- Galaxy shape. We use stellar morphology to define galaxy shape via two parameters: the minor-to-major axis ratio (s) and the middle-to-major axis ratio (q). Following Pillepich et al. (2019) (see their Figure 8) we define disk galaxies as those that satisfy two criteria: a minor-to-major ratio $s<0.35$ and a middle-to-major ratio $q>0.65$; otherwise, galaxies are classified as non-disks.

- SMBH kinetic feedback energy. For each galaxy, $E_{\mathrm{kin}}$ is defined as the accumulated feedback energy ever released by its central $\mathrm{SMBH}$ while in the kinetic mode.

To derive the gas properties we select all non star-forming gas cells that belong to a given halo, as identified by the FoF algorithm. As a result, we include not only gas cells that are gravitationally bound to the central galaxy, but also those that belong to the halo's satellites as well as gas in winds and outflows that are not necessarily gravitationally bound to galaxies. This best mimics observations where it is generally non-trivial to separate e.g. X-ray emission arising from satellites or from high-velocity outflows. In addition to density, temperature, and metallicity, which are direct simulation outputs and that determine the X-ray luminosity of the volume-filling gas, we also explicitly quantify:

- Gas pressure: $P_{\mathrm{e}}=n_{\mathrm{e}} k_{\mathrm{B}} T$, where $n_{\mathrm{e}}$ is the number density of electrons, and $T$ is the temperature of the gas cell.

- Gas entropy: $K=k_{\mathrm{B}} T / n_{\mathrm{e}}^{2 / 3}$.

- X-ray quantities: For most of the theoretical quantification provided in this paper, we compute the intrinsic X-ray emission on a cell-by-cell basis, starting from the gas density, temperature, and metal abundances predicted by the simulations and using the emission model APEC from XSPEC (Smith et al. 2001). Namely, for the results of Figures 7, 8, A1, and A3, we compute intrinsic Xray emission and do not mock observational effects such as galactic absorption or instrumental response. However, to make actual predictions for X-ray observations (i.e. in Section 5.3 and Figure 9), we also create mock photon counts as observed with the eROSITA telescope (Merloni et al. 2012). For this task we employ the APEC model plus galactic absorption (i.e. ['wabs(apec)']) with a column density of $\mathrm{n}_{\mathrm{H}}=10^{20} \mathrm{~cm}^{-2}$. The mock spectrum, i.e. photon counts as a function of energy, is generated via the fakeit procedure implemented in XSPEC taking into account the eROSITA instrument response files ${ }^{1}$.

\subsection{Edge-on projections}

Throughout the paper, we analyze galaxies and their CGM in edgeon projections. We first rotate each galaxy edge-on, then we create a 2D map of gas properties for all gas within a cube of size $2 \times R_{200 c}$ centered on the point with minimum gravitational potential. The edge-on rotation is based on the stellar minor axis of a galaxy, chosen as the total stellar angular momentum of stars within twice the stellarhalf mass radius. Our fiducial map resolution is $30 \times 30$ pixels, which always has a fixed size in units of the virial radius, and each pixel has the same fraction size of $R_{200 c}$. For reference, in our lowest (highest) mass bin, $M_{*} \sim 10^{10.0} M_{\odot}$ and $R_{200 \mathrm{c}} \sim 100 \mathrm{kpc}\left(M_{*} \sim 10^{11.4} M_{\odot}\right.$ and $\left.R_{200 \mathrm{c}} \sim 570 \mathrm{kpc}\right)$, the size of each pixel is $\sim 6(\sim 40) \mathrm{kpc}$. For the projection, gas column densities and X-ray fluxes are summed over gas along the line of sight, while other averaged quantities, e.g. gas temperature and metallicity, are taken as the mass-weighted mean of the gas cells along the line of sight. We also examine the X-ray emission-weighted averages of the gas temperature and metallicity and present the results in Appendix B.

\subsection{Stacked maps}

For stacked maps, the individual galaxy maps are combined pixelby-pixel via median stacking. We discarded either mean or mode stacking as they emphasize the existence of sub-structure (i.e. satellite galaxies) that exist in only one halo of the stack, confusing our study of the average properties of the diffuse CGM gas.

\subsection{Characterization of the CGM anisotropy}

Throughout the paper, the angular location of CGM gas is defined with respect to the stellar minor axis of galaxies (Section 2.3), so that an azimuthal angle of 0 degrees denotes alignment with a galaxy's major axis, whereas an azimuthal angle of 90 degrees signifies alignment with the minor axis. We make no distinction among the four quadrants of a galaxy's edge-on projection, which are then "stacked" together, and a galaxy does not need to be late-type or disk-shaped for a minor or major axis to be defined.

To characterize the level of anisotropy of the CGM, we use the ratio between properties measured along the minor versus major axes at a given galactocentric distance. Starting from the edge-on projected maps of galaxies, either individual or stacked (Sections 2.3 and 2.4), we measure the minor (major) axis value for gas at azimuthal angles, in each quadrant, of $>81(<9)$ degrees and within a thin shell of $0.04 R_{200 \mathrm{c}}$ thickness. We label the ratio of these two values the "minor-to-major ratio" for CGM properties such as density, temperature, and metallicity. A minor-to-major ratio close to 0 (in the $\log$ )

\footnotetext{
1 https://wiki.mpe.mpg.de/eRosita/erocalib_calibration
} 
denotes isotropy; a minor-to-major ratio larger (smaller) than 0 indicates that a give property is enhanced (suppressed) along the minor axis. We measure the minor-to-major metric on a galaxy-by-galaxy basis. Namely, we start from individual galaxy maps and record the minor-to-major ratio of the gas properties for each, at various galactocentric distances. Results for galaxy samples are given in terms of the median ratios across the considered galaxies, and galaxy-to-galaxy variation is represented by e.g. the 32-th and 68-th percentiles of the ratio distribution. In all plots, shaded areas represent the locus of such percentiles, i.e. the $\sigma / 2$ galaxy-to-galaxy scatter in the anisotropy. Note that in selected instances, where noted, we will comment on the median minor-to-major ratios that are obtained instead from stacked images of galaxy subsamples.

\subsection{Illustris and EAGLE Simulations}

We compare TNG100 to two other publicly-available cosmological simulations: the original Illustris simulation and EAGLE, which both have comparable simulated volumes and mass resolutions.

- Illustris. We use the Illustris simulation (Vogelsberger et al. 2014a,b; Genel et al. 2014; Sijacki et al. 2015), which is the predecessor of IllustrisTNG. Illustris is a series of large-scale hydrodynamical simulations, which were also run with AREPO code (Springel 2010). We use the highest resolution Illustris-1 run, which simulates a volume of $(106.5 \mathrm{Mpc})^{3}$ with baryon mass resolution $m_{\text {baryon }}=1.26 \times 10^{6} M_{\odot}$. The Illustris model of galaxy formation includes radiative cooling (both primordial and metal-line cooling); star formation, stellar evolution, metal enrichment, and stellar feedback from supernovae; SMBHs seeding, growth, as well as their feedback in radio, quasar, and radiative modes. A detailed description of the model is provided in Vogelsberger et al. (2013).

- EAGLE. We also use the EAGLE simulation (Schaye et al. 2015; Crain et al. 2015). The EAGLE project is a suite of cosmological hydrodynamical simulations of various simulated volumes and resolutions. For our study, we make use of the flagship run, referred as 'Ref-L0100N1504', which simulates a cosmic box with a comoving side-length of $100 \mathrm{Mpc}$ and a baryon mass resolution of $m_{\text {baryon }}=1.81 \times 10^{6} M_{\odot}($ McAlpine et al. 2016). The EAGLE simulations are performed with a modified version of the GADGET-3 smoothed particle hydrodynamics (SPH) code (Springel 2005). They implement a wide range of physical processes including radiative cooling and photoheating, star formation, stellar mass-loss and thermal stellar feedback; procedures for SMBH seeding, growth, as well as SMBH thermal feedback, which we discuss in more detail later. We refer to Schaye et al. (2015) for the EAGLE model description.

\section{ANGULAR DEPENDENCE OF CGM PROPERTIES}

\subsection{CGM anisotropy according to IllustrisTNG}

Figure 1 visualizes how the CGM properties of TNG galaxies at $z=0$ vary with angular location. Results are given for a subsample of TNG100 central massive galaxies with stellar mass $M_{*}=10^{11 \pm 0.1} M_{\odot}$. The top row presents stacked maps of gas thermodynamical properties; i.e. gas column density $\left(\Sigma_{\text {gas }}\right)$, mass-weighted temperature $\left(T_{\mathrm{mw}}\right)$, and mass-weighted metallicity $\left(Z_{\mathrm{mw}}\right)$. The maps are shown edge-on, with the vertical direction aligned along the minor axis of galaxies (i.e. with respect to their stellar angular momentum - see Section 2.4), whereas the horizontal direction lies on the same plane of the galactic "disks". To best visualize the spatially anisotropic distribution of the CGM properties, we then normalize each quantity with respect to its azimuthal average (bottom row), where brighter colors indicate an enhancement of the gas properties with respect to their azimuthal average.

To quantify these trends, Figure 2 shows how the gas properties within a projected, thin shell at a certain galactocentric distance vary as a function of azimuthal angle. The azimuthal dependence is computed from individual galaxy maps, and the trends represent the population median behavior, with shaded areas denoting the $\sim \sigma / 2$ intrinsic galaxy-to-galaxy scatter.

Figures 1 and 2 show that the TNG model predicts that the CGM of massive galaxies is anisotropic. This anisotropy arises with different dependencies, and to different degrees, for different gas properties. The CGM gas density is lower along the minor axis of galaxies, whereas gas temperature and metallicity are higher. Quantitatively, the amplitude of the anisotropy is largest for gas density and metallicity, reaching on average $\sim 0.1 \mathrm{dex}(\sim 25 \%)$, although these two trend with azimuthal angle are in opposite directions. Gas temperature varies with angle less strongly, by $\sim 0.05 \mathrm{dex}(\sim 12 \%)$.

The spatial anisotropies extend from the central regions of haloes $\left(r \sim 0.25 R_{200 \mathrm{c}}\right)$ all the way to the canonical halo boundaries $\left(r \sim R_{200 \mathrm{c}}\right)$. According to TNG, the relative anisotropy of gas and temperature does not depend on galactocentric distance, at this mass, whereas the angular dependence of the gas metallicity is stronger at larger distances. We note that the galaxy-to-galaxy scatter is comparable or even larger than the amplitude of the signals, hinting that the degree of anisotropy may depend on further galaxy properties beyond mass. For the sake of clarity, we opt to report the intrinsic scatter in term of the interval between the 32th and 68th percentile elements.

In TNG, the relative under-density of CGM gas along the minor axis of $\sim 10^{11} M_{\odot}$ galaxies - with these regions being simultaneously also hotter and more metal-enriched - is a consequence of galactic outflows. Although the energy injection from both stellar and SMBH feedback is isotropic in the TNG model, galactic-scale outflows tend to emerge along paths of least resistance creating bipolar structures oriented along the minor axis of galaxies (Nelson et al. 2019b). When advancing to larger galactocentric altitudes, such outflows push gas out of the central regions of haloes (Terrazas et al. 2020; Zinger et al. 2020; Davies et al. 2020) while at the same time thermalizing and heating up the gas via shocks (Weinberger et al. 2017; Pillepich et al. 2021). Metals that have been previously produced in the central star-forming regions of galaxies are carried by outflows, thereby enriching the gas above and below the galactic disks (Pillepich et al. 2021). The radial dependence of the CGM metallicity anisotropy can be explained by the changing contrast of metal-poor gas inflows, preferentially along the galactic planes, and metal-rich outflows (Péroux et al. 2020).

\subsection{Mass trends of the CGM anisotropy}

We proceed to quantify how the CGM anisotropy varies with galaxy mass, for central galaxies with stellar mass larger than $M_{*} \sim 10^{10} M_{\odot}$ (see Section 2.2), using the minor-to-major ratio, which is derived from individual galaxy maps as described in Section 2.5.

Figure 3 shows the level of anisotropy of gas density (first row), temperature (second row), and metallicity (third row) as a function of various masses: galaxy stellar mass ( $M_{*}$, first column), total halo mass $\left(M_{200 \mathrm{c}}\right.$, second column), and SMBH mass $\left(M_{\mathrm{BH}}\right.$, third column $)$, at three galactocentric distances.

According to the TNG model, the anisotropy of gas density and 


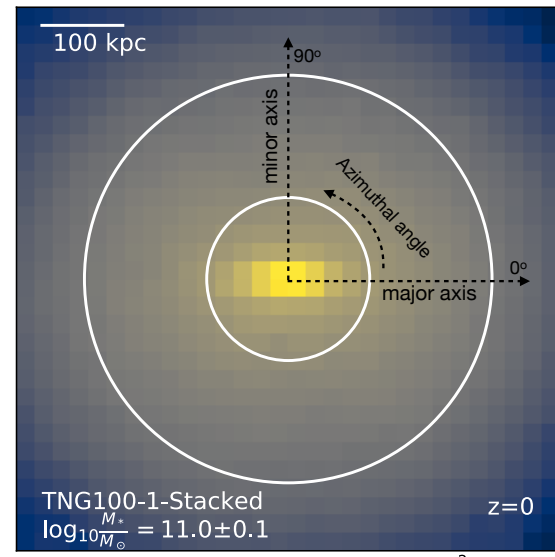

Gas Column Density $\left[\log _{10} \mathrm{M}_{\odot} \mathrm{kpc}^{-2}\right]$

4.8

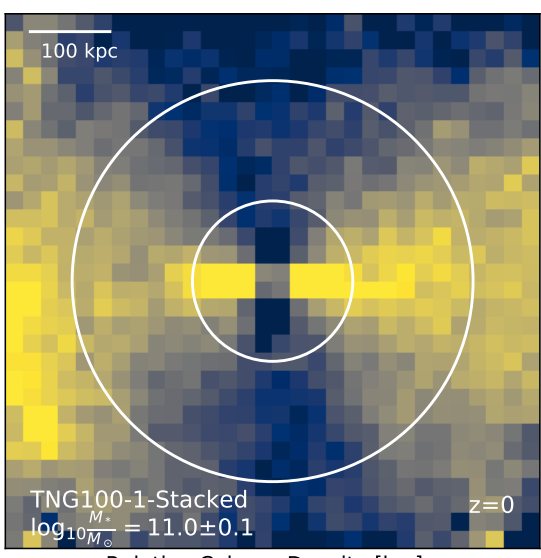

Relative Column Density [log]

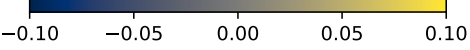

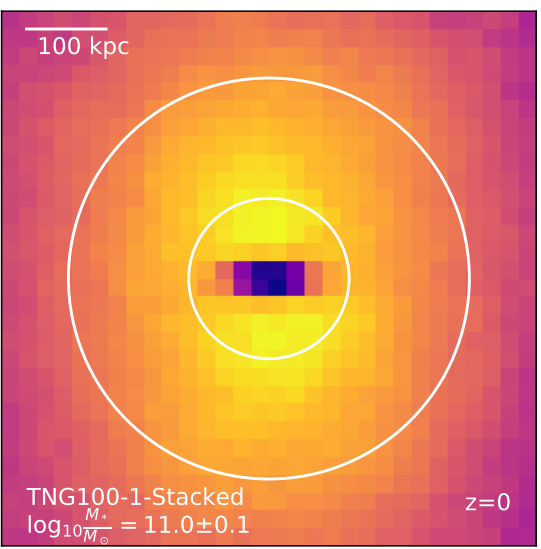

Temperature $\left[\log _{10} \mathrm{~K}\right]$
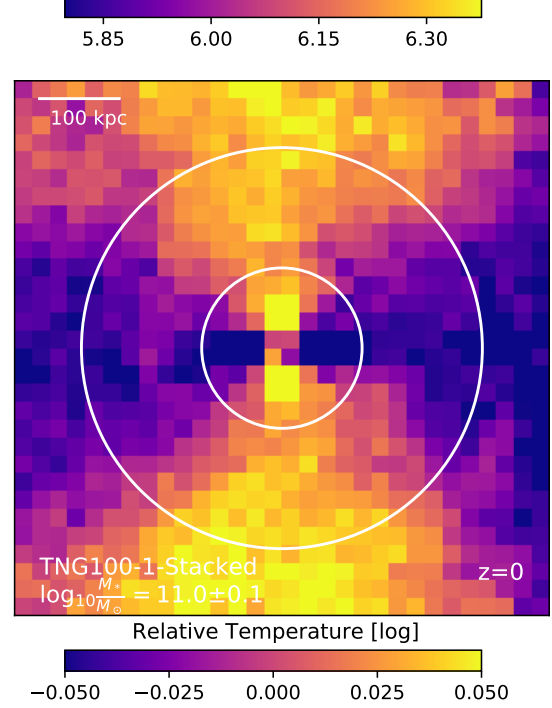

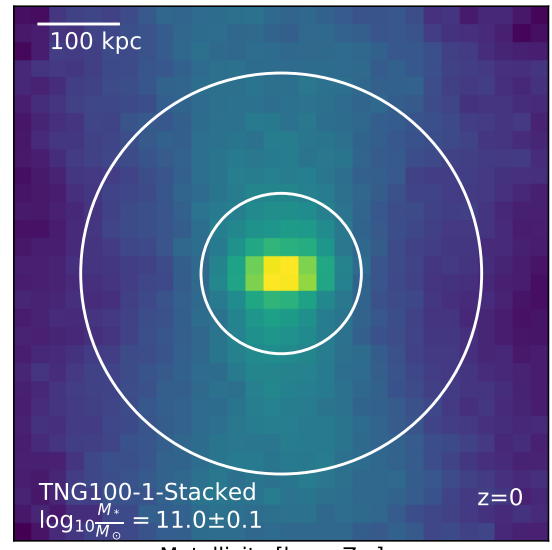

$\begin{aligned} \log _{10} \bar{M}_{\odot} & =11.0 \pm 0.1 \\ & \text { Metallicity }\left[\log _{10} Z_{\odot}\right]\end{aligned}$
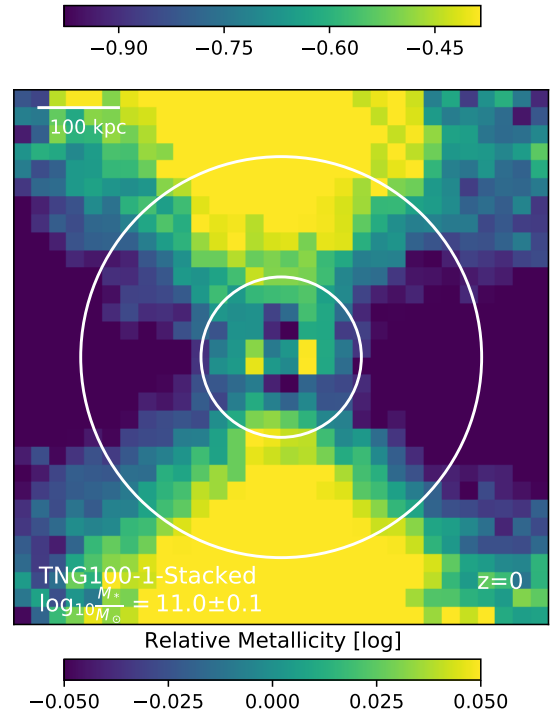

Figure 1. Anisotropy of the CGM properties according to the TNG model at $z=0$. The results are obtained by stacking 277 central galaxies with $M_{*}=$ $10^{11.0 \pm 0.1} M_{\odot}$ from TNG100 (see Sections 2.3 and 2.4 for details). Top row: Stacked maps of gas properties: from left to right, gas column density, gas temperature, and gas metallicity. The stacks are made for edge-on galaxies, rotated based on their stellar component, and the maps span a cubic region of $2 R_{200 \mathrm{c}}$ per side. The two concentric circles show galactocentric distances of $0.25 R_{200 \mathrm{c}}$ and $0.75 R_{200 \mathrm{c}}$. Bottom row: Relative stacked maps, whereby the gas properties are normalized to their azimuthally-averaged value as a function of distance. Brighter colors indicate an enhancement of the gas properties relative to their azimuthal average. Massive galaxies have angle-dependent CGM properties: the gas is less dense, hotter, and more enriched along the minor-axis directions.
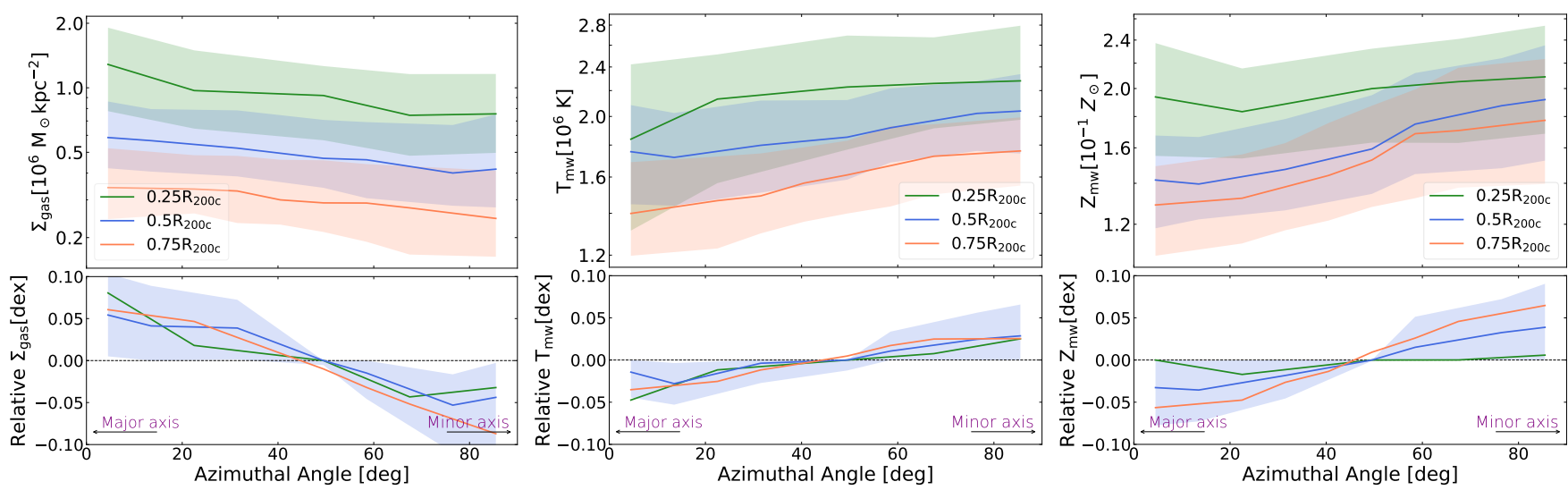

Figure 2. Quantification of the CGM anisotropy predicted by TNG100 and visualized in Figure 1. We show gas properties as a function of azimuthal angle at varying galactocentric distances (top) and the corresponding relative properties with respect to their azimuthal median (bottom), for TNG100 galaxies at $z=0$ with $M_{*}=10^{11.0 \pm 0.1} M_{\odot}$. The solid lines are the population median, which are derived from individual galaxy maps, while the shaded areas specify the 32 -th and 68-th percentiles; i.e. $\sim \sigma / 2$ galaxy-to-galaxy intrinsic variation. TNG predicts an angular dependence for CGM properties in massive galaxies, with the gas being more diluted, hotter, and more chemically enriched along the minor axes. 

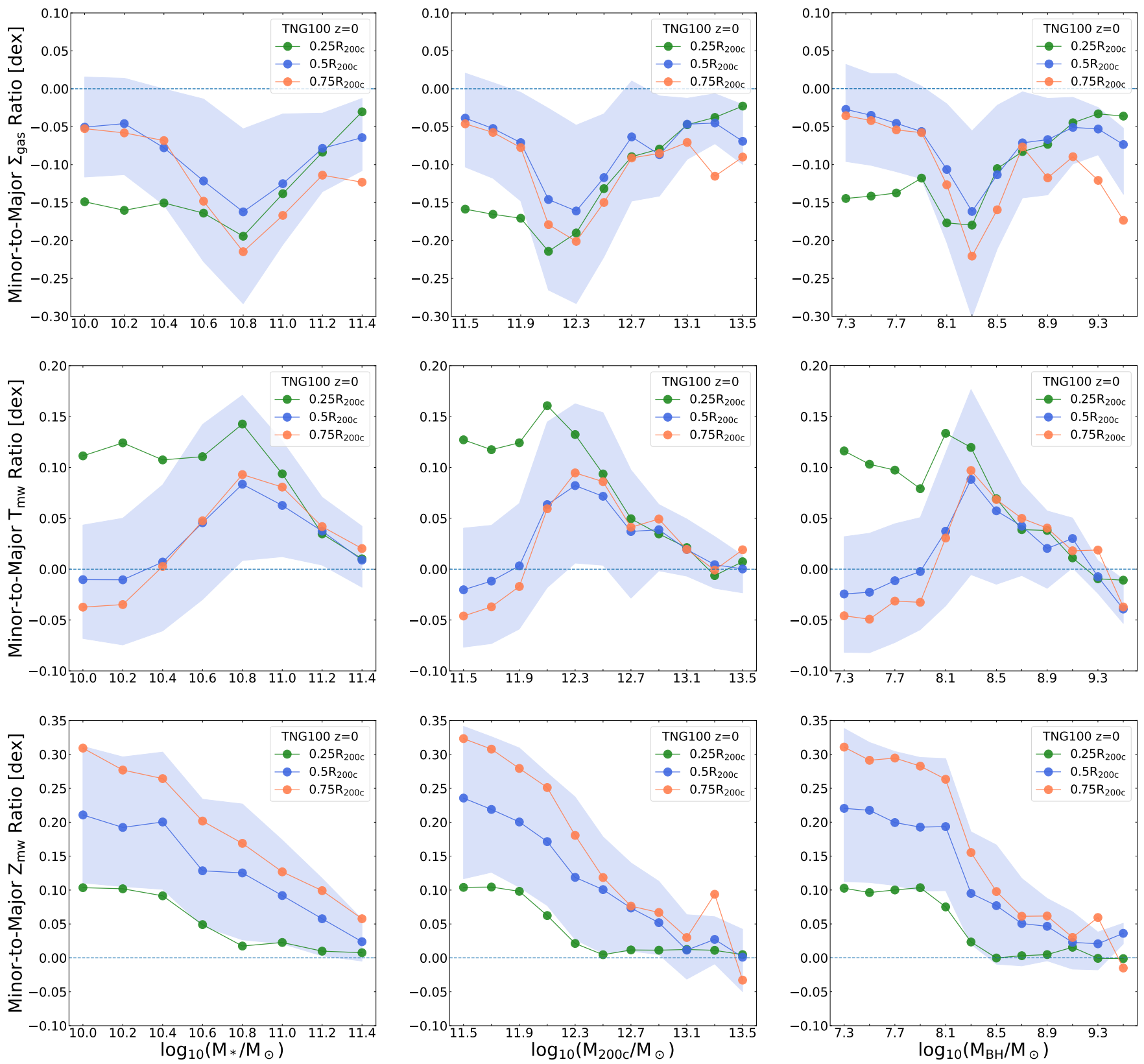

Figure 3. Mass dependence of the CGM anisotropy for TNG100 galaxies at $z=0$, quantified with the minor-to-major ratio (see Section 2.5) of galaxies in a given mass bin, measured at various galactocentric distances. From top to bottom, we show results for gas column density, mass-weighted gas temperature, and mass-weighted gas metallicity. Different columns give the dependence on different masses: galaxy stellar mass $\left(M_{*}\right.$, left $)$, total halo mass $\left(M_{200 c}\right.$, middle $)$, and SMBH mass $\left(M_{\mathrm{BH}}, r i g h t\right)$. The symbols and solid lines represent the galaxy-population median, whereas the grey shaded area is the 32th-to-68th percentile interval (i.e. $\sim \sigma / 2$ ) for the measurement at $r=0.5 R_{200}$ c i.e. representing the galaxy-to-galaxy variation. According to TNG, the CGM anisotropy of gas density and gas temperature is maximal at the transitional mass scale of $M_{*} \simeq 10^{10.5-11} M_{\odot}$, i.e. $M_{200 \mathrm{c}} \simeq 10^{12.1-12.5} M_{\odot}$ and $M_{\mathrm{BH}} \simeq 10^{8.0-8.5} M_{\odot}$.

temperature is non-monotonic with mass: the angular modulation increases with mass at the low-mass end, peaks at a maximum, and then decreases with mass at the high-mass end, irrespective of galactocentric distance. The anisotropy is maximized at the same galaxy stellar mass for both density and temperature, $M_{*} \sim 10^{10.5-11} M_{\odot}$. However, as shown in the previous Section, the gas density and temperature display anisotropic signals in opposite directions, and this pattern is consistent across the considered mass range. For instance, at the peak of CGM anisotropy, TNG galaxies exhibit under-dense halo gas along the minor axis (by $\sim 0.15-0.20 \mathrm{dex}$ ), whereas the gas temperature is higher (by $\sim 0.05-0.15 \mathrm{dex}$ ). The level of density and temperature angular dependence is roughly distance independent at the high-mass end (consistent with the results of Figure 1 for $M_{*} \sim 10^{11} M_{\odot}$ galaxies), whereas at the low-mass end gas density and temperature are more anisotropic at regions closer to the galaxies center (see e.g. the green curves at $r \sim 0.25 R_{200 \mathrm{c}}$ ). On the other hand, the anisotropy of the CGM metallicity is strongest at the low-mass end $\left(M_{*} \sim 10^{10} M_{\odot}\right)$, where gas metallicities vary by $0.1-0.3$ dex between directions along the minor vs. major axis, before monotonically decreasing for more massive systems. The radial dependence of the metallicity anisotropy, as found in the previous section at the mass bin of $M_{*} \sim 10^{11} M_{\odot}$, is preserved across the 


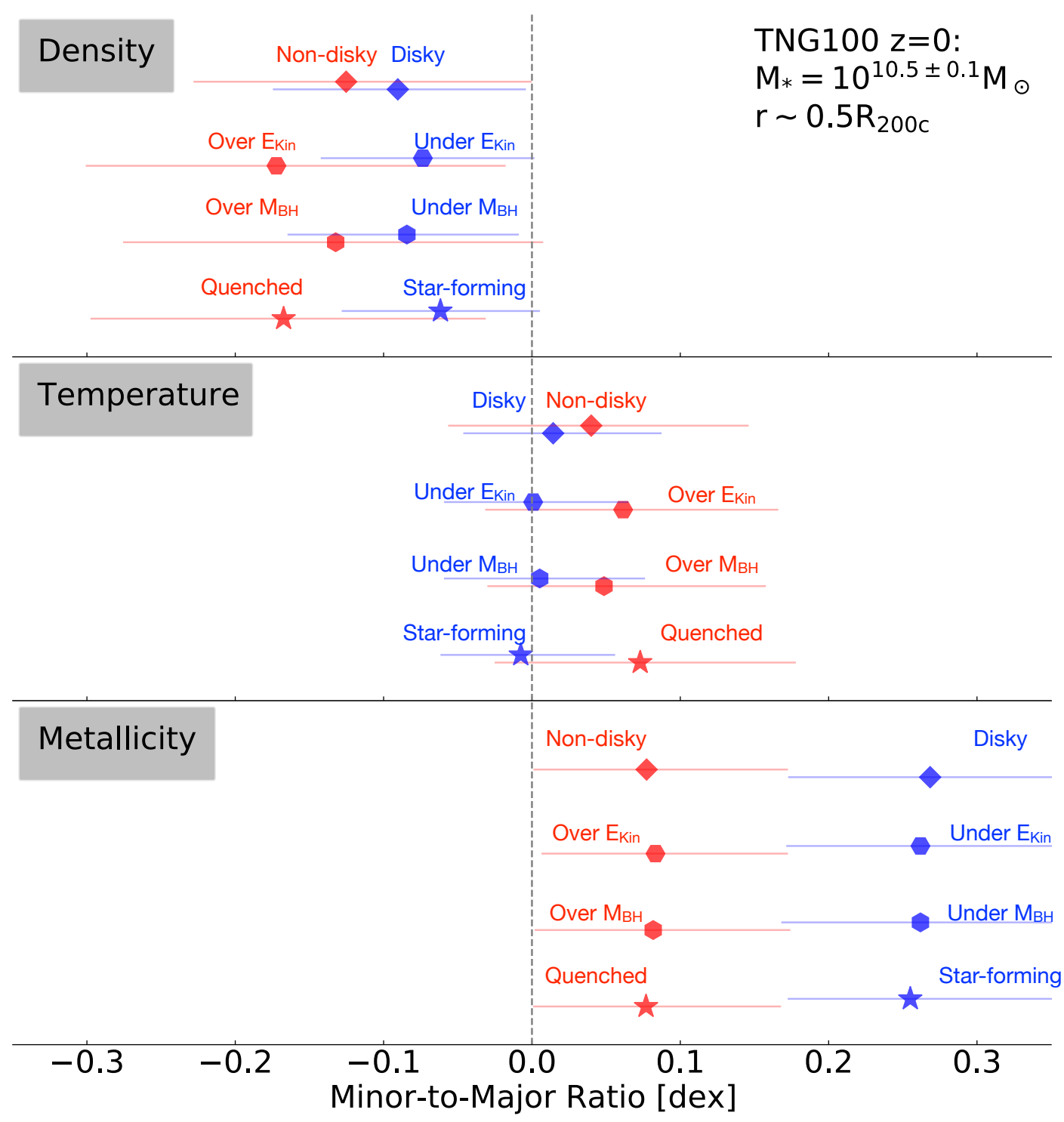

Figure 4. Dependence of the CGM anisotropy on various galaxy properties according to the TNG model. From top to bottom, we give results for $\Sigma_{\text {gas }}, T_{\mathrm{mw}}$, and $Z_{\mathrm{mw}}$, respectively. The considered galaxy properties are: star formation status, SMBH mass, accumulated energy in SMBH kinetic feedback ( $\left.\mathrm{E}_{\mathrm{kin}}\right)$, and galaxy stellar morphology. The star formation status of a galaxy is defined based on its distance from the star-forming main sequence, whereas for $M_{\mathrm{BH}}$ and $E_{\mathrm{kin}}$, the 'under' and 'over' quantities are defined relative to the median values in the considered mass range (see Sections 2.2 and 3.3 for details). Here we show the median results from $742 M_{*} \sim 10^{10.5} M_{\odot}$ TNG100 galaxies (filled, large symbols), at the galactocentric distance $r \sim 0.5 R_{200 c}$. The error bars represent $\sim \sigma / 2$ galaxy-to-galaxy variation. According to the TNG model, massive galaxies that are quenched, host over-massive SMBHs, and/or whose SMBHs have injected relatively more kinetic feedback energy, exhibit stronger angular modulations of their CGM density and temperature than e.g. their star-forming counterparts. These trends are reversed for the metallicity anisotropy.

considered mass range, with stronger angular variations at larger galactocentric distances, and more so around low-mass galaxies.

For gas density and temperature, the galaxy stellar mass at which the CGM anisotropy is maximal $\left(M_{*} \sim 10^{10.5-11} M_{\odot}\right.$, left column of Figure 3) corresponds to total halo masses of $M_{200 c} \sim$ $10^{12.1-12.5} M_{\odot}$ and SMBH masses of $M_{\mathrm{BH}} \sim 10^{8.0-8.5} M_{\odot}(\mathrm{mid}$ and right columns Figure 3, respectively). This suggests that the CGM anisotropy may be connected to SMBH feedback activity; i.e. to SMBH-driven outflows, as this SMBH-mass range corresponds to the scale at which, in the TNG model, SMBHs typically switch from the thermal to the kinetic feedback mode (Weinberger et al. $2017)$ and to stellar mass scales $\left(M_{*} \sim 10^{10.5-11} M_{\odot}\right)$ above which SMBH kinetic feedback becomes the dominant channel in galax- ies at low redshifts (Weinberger et al. 2018). We expand on the connection between CGM anisotropy and SMBH feedback activity in the next Section. It is worth mentioning that the distribution of both CGM density and temperature, except for the measurements at small radii $\left(r \sim 0.25 R_{200 \mathrm{c}}\right)$, is more isotropic at the both lowmass and high-mass ends. We speculate that the CGM anisotropy is most prominent at the transitional mass range around the Milky Way's mass because here two physical conditions are in place: i) the occurrence of sufficiently-strong, feedback-driven outflows; ii) the presence of a gaseous disk in the inner regions of galaxies that can (re)direct outflows in bi-polar directions. At higher masses, outflows become more isotropic in the absence of such a gaseous disk; towards the lower-mass end ( $\lesssim M_{*} \sim 10^{10} M_{\odot}$ ), where outflows are driven 
predominantly by stellar feedback and by SMBHs in thermal mode, they appear to be ineffective at large galactocentric distances (see also Section 6.2 in Pillepich et al. 2021 for a relevant discussion).

On the other hand, we speculate that the different trends for the metallicity anisotropy could be explained by the decrease of metal content of massive galaxies. Torrey et al. (2019) showed that the gas-phase metallicity-stellar mass relation in the TNG simulations flattens around $M_{*} \sim 10^{10.5} M_{\odot}$ and declines towards the high-mass end due to the suppression of star formation. As a consequence, the gas carried out by outflows in more massive galaxies may be less metal rich than that removed from the central regions of less-massive and more star forming galaxies (and progenitors). Furthermore, the gaseous halo in massive galaxies is already enriched at early times and it is likely more well-mixed compared to low-mass galaxies. As a consequence, for massive galaxies the CGM metallicity along the minor axis is more similar to that of the azimuthally-averaged halo gas, even at a mass range where outflows are strongest.

\subsection{Connection to SMBH activity}

What is the physical cause for the CGM anisotropy around TNG galaxies, specifically massive ones? In Figure 4, we show the dependence of the angular modulation at $\sim 0.5 R_{200 \mathrm{c}}$ on various galaxy properties. We consider the CGM density (top row), temperature (middle row), and metallicity (bottom row), for $z=0$ TNG galaxies with $M_{*}=10^{10.5 \pm 0.1} M_{\odot}$. We choose this stellar mass bin because at this mass scale, both in observations and in the TNG simulations (see e.g. Donnari et al. 2020), the number of star-forming and quenched galaxies is comparable, i.e. about 370 galaxies in each category in TNG100. For each galaxy property, we subdivide the sample into two subgroups based on:

- Star formation status: quenched vs. star-forming (Section 2.1).

- SMBH mass: over-massive vs. under-massive $M_{\mathrm{BH}}$ relative to the median $M_{\mathrm{BH}}$ value.

- $\mathrm{SMBH}$ accumulated kinetic feedback $\left(\mathrm{E}_{\text {kin }}\right)$ : over-energetic vs. under-energetic $\mathrm{E}_{\mathrm{kin}}$ relative to the median $\mathrm{E}_{\mathrm{kin}}$ value.

- Galaxy shape: disky vs. non-disky, as defined in Section 2.1.

For each CGM property, we evaluate the anisotropy from the gas maps on a galaxy-by-galaxy basis; i.e. without stacking. We display the median minor-to-major ratio of the sample (large symbols in Figure 4) while the error bars represent the galaxy-to-galaxy variation across the 32th and 68th percentiles of the considered galaxy sample.

First, we consider the CGM temperature anisotropy and its dependence on the galaxy star formation status. It has been extensively shown that in TNG the star formation in massive galaxies is quenched due to ejective effect of the SMBH kinetic feedback (Weinberger et al. 2017, 2018; Nelson et al. 2018a; Terrazas et al. 2020; Davies et al. 2020). Therefore, quiescence can be considered as a proxy for the (past) action of SMBH feedback. As visible in the middle row of Figure 4 , at the fixed galaxy stellar mass of $\sim 10^{10.5} M_{\odot}$, quenched galaxies exhibit systematically elevated minor-to-major $T_{\mathrm{mw}}$ ratios $(\sim 0.1 \mathrm{dex})$ compared to the star-forming counterparts: for the latter, in fact, the gas temperature is, on average, isotropic - minor-tomajor temperature ratio $\simeq 0$. In terms of the impact of SMBHs, galaxies with over-massive SMBHs ("over $M_{\mathrm{BH}}$ ") exhibit a similar level of temperature anisotropy, whereas those with under-massive SMBHs ("under $M_{\mathrm{BH}}$ ") show on average no temperature angular modulation. Upon closer inspection of the subsamples, we notice that for galaxies with over $M_{\mathrm{BH}}$, at this mass range, the vast majority of their SMBHs $(\approx 90 \%)$ have already switched to the kinetic mode, whereas, in the under $M_{\mathrm{BH}}$ subsample, most of the SMBHs
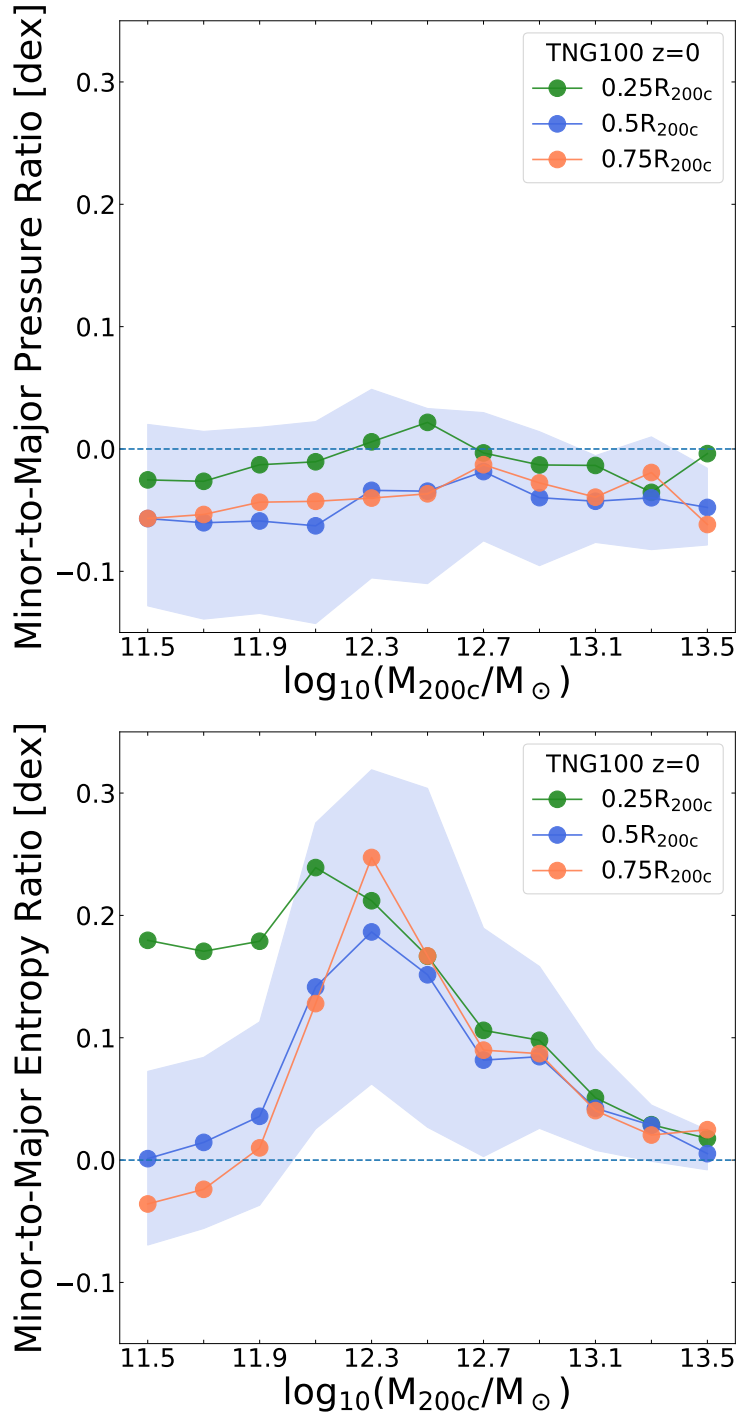

Figure 5. CGM anisotropy in mass-weighted gas pressure (top) and gas entropy (bottom) for TNG100 galaxies at $z=0$. The symbols and solid lines represent the median minor-to-major ratio of galaxies in a given mass bin, whereas the grey shaded area shows the 32th to 68th percentiles (i.e. $\sim \sigma / 2$ ) for the $r=0.5 R_{200}$ c curve. The CGM entropy captures in full the angular modulations of the underlying gas properties (i.e. density and temperature), whereas pressure is essentially isotropic.

( $\gtrsim 60 \%$ ) are still in the thermal mode. By inspecting the variations across galaxies with different levels of SMBH feedback energy ever released in SMBH-driven winds, we find that galaxies with aboveaverage $E_{\text {kin }}$ exhibit more pronounced temperature anisotropies. Interestingly, considering the dependences on stellar morphology, we find that the non-disky subsample appears to have only marginally stronger anisotropy in $T_{\mathrm{mw}}$. Namely, the distinction between the two morphologically-defined subsamples is less significant than that associated to SMBH-related diagnostics.

The level of anisotropy of the CGM density depends on the considered galaxy properties similarly as for gas temperature. Namely, the angular modulation of the gas density is stronger in quenched galaxies, galaxies with over massive SMBHs or larger-than average amounts of energy injected via SMBH-driven winds. The anisotropy 
appears less sensitive to the stellar morphology, though it is slightly stronger in non-disky galaxies.

On the other hand, the CGM metallicity anisotropy exhibits opposite trends with galaxy and SMBH properties in comparison to gas density and temperature. The angular dependence of the gas metallicity is stronger in star-forming, disky galaxies with relatively undermassive $\mathrm{SMBHs}$ and low $\mathrm{E}_{\mathrm{kin}}$ values. This is consistent with the the stronger CGM metallicity anisotropy in low-mass star-forming galaxies (Figure 3), where galactic outflows driven by stellar feedback e.g. supernovae explosions are: a) dominant over those from SMBH activity (Nelson et al. 2019b); and b) able to enrich the CGM with metals originating from the inner regions of galaxies. Stellar feedback has been interpreted as the physical driver of the angular dependence of gas metallicity for $M_{*} \lesssim 10^{10.5} M_{\odot}$ in TNG50 (Péroux et al. 2020).

Overall our picture is that, in the TNG model, SMBH-driven outflows in the kinetic, low-accretion mode produce the observed CGM anisotropies in gas density and temperature at the high-mass end $\left(M_{*} \gtrsim 10^{10.5-11} M_{\odot}\right)$. This does not exclude the possibility that the angular modulation of CGM density and temperature within the central regions of haloes $\left(r \sim 0.25 R_{200 \mathrm{c}}\right)$, as well as the metallicity anisotropy at all radii, of lower-mass systems may be caused by other feedback mechanisms: i.e. stellar feedback and SMBH feedback in the thermal mode (Weinberger et al. 2018).

\subsection{CGM anisotropy in pressure and entropy}

Figure 5 explores whether the anisotropic nature of the CGM density, temperature, and metallicity is imprinted in two other important thermodynamical properties: gas pressure and gas entropy, as defined in Section 2.2. We show the median minor-to-major ratios of galaxies in bins of halo mass, for mass-weighted gas pressure $\left(P_{\mathrm{mw}}\right.$, top $)$ and mass-weighted gas entropy $\left(K_{\mathrm{mw}}\right.$, bottom $)$, at the same three galactocentric distances as previously.

The gas pressure exhibits only minor differences between minor vs. major axes values ( $\lesssim 0.05 \mathrm{dex})$ across the whole inspected mass range. This result is a direct consequence of the gas pressure being effectively the product of density and temperature: the two quantities depend on azimuthal angle in opposite directions, as shown in Section 3.2, and these trends largely cancel out. On the other hand, the entropy of the CGM exhibits a similar anisotropy, and associated mass dependence, as the gas temperature. Namely, entropy is enhanced along the minor axis of galaxies, and this anisotropy is maximized for galaxies in $M_{200 c} \sim 10^{11.9-12.5} M_{\odot}$ haloes; i.e. the mass scale where galaxies transition from star-forming to quiescent. In fact, the angular modulation of entropy is the largest of all thermodynamical properties studied thus far, as it encodes information on both gas density ( $\left.\propto n_{\mathrm{e}}^{-2 / 3}\right)$ and temperature $(\propto T)$.

As shown by Zinger et al. (2020), gas entropy is a sensitive diagnostic of feedback injection and a good proxy for quiescence, with quiescent galaxies exhibiting much larger entropy, in both their ISM and CGM, than their star-forming counterparts. In TNG this is due to the ejection and heating of gas via SMBH-driven winds. Our analysis shows that the entropy's increase due to SMBH feedback occurs preferentially along the minor axis of galaxies.

\section{COMPARISON OF COSMOLOGICAL SIMULATIONS}

We have shown thus far that, in the TNG simulations, feedback from stars and SMBHs imprints its effect on the CGM in a rather unique way: by making gas density, temperature, metallicity, and
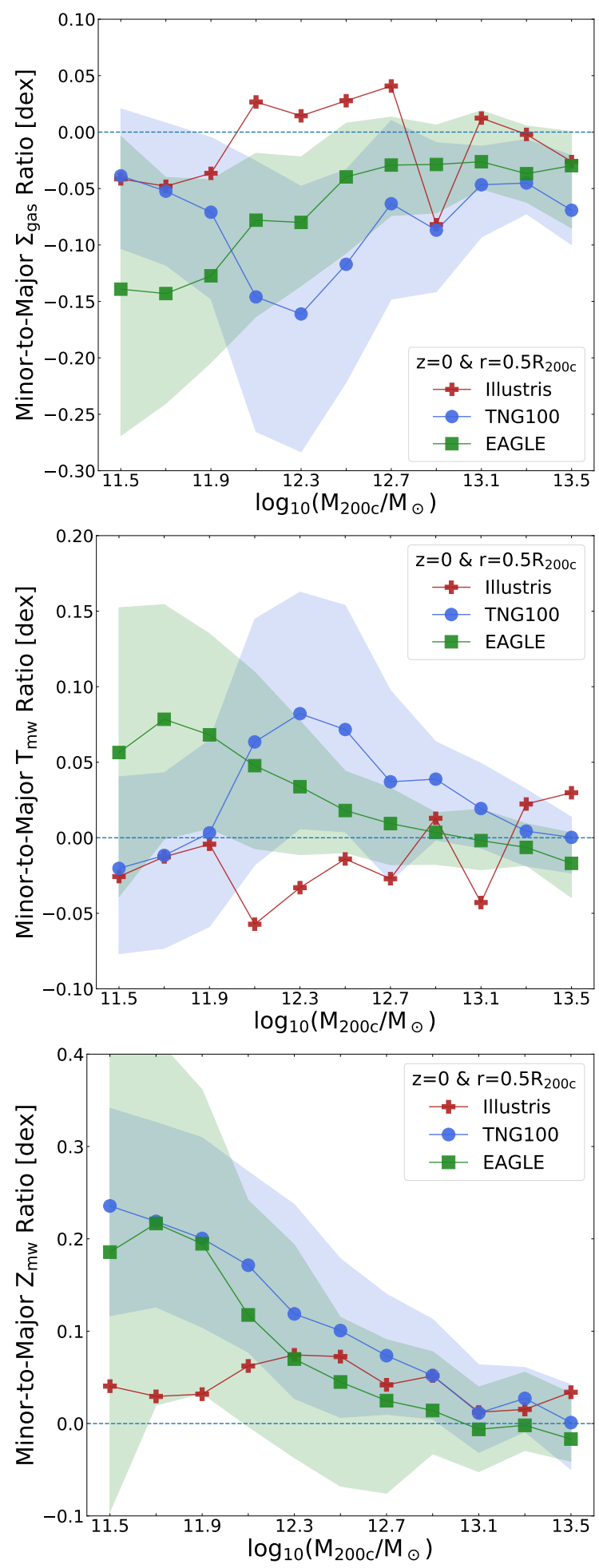

Figure 6. The CGM anisotropy according to the Illustris, TNG, and EAGLE simulations, in comparison. From top to bottom, we show results for gas column density, mass-weighted gas temperature, and mass-weighted gas metallicity, as a function of total halo mass. The minor-to-major ratios are measured for simulated galaxies at $z=0$ and at a galactocentric distance of $r=0.5 R_{200}$. For clarity, we show the scatter only for the TNG and EAGLE simulations. Illustris predicts weaker CGM anisotropy signals than EAGLE and TNG, and the latter two models agree qualitatively but not quantitatively, with generally lower densities, higher temperatures, and higher metallicities in the CGM along the minor axes of galaxies in both models. 
Table 1. Summary of stellar and SMBH feedback models in three simulations: Illustris, TNG, and EAGLE. For the sake of brevity, we list only main features of the models (locality, stochasticity, form of feedback energy and orientation) and refer to the main text and references therein for expanded descriptions.

\begin{tabular}{|c|c|c|}
\hline Illustris & TNG & EAGLE \\
\hline $\begin{array}{l}\text { Stellar feedback } \\
\text { - Non-local: temporary hydro decoupling } \\
\text { - Timing: continuous probabilistic, } \propto \text { SFR } \\
\text { - Feedback energy: kinetic only (cold wind) } \\
\text { - Orientation: bi-polar }\end{array}$ & $\begin{array}{l}\text { - Non-local: temporary hydro decoupling } \\
\text { - Timing: continuous probabilistic, } \propto \text { SFR } \\
\text { - Feedback energy: kinetic + thermal (warm) } \\
\text { - Orientation: isotropic }\end{array}$ & $\begin{array}{l}\text { - Local: direct ISM heating } \\
\text { - Timing: stochastic probabilistic, } \Delta T=10^{7.5} K \\
\text { - Feedback energy: thermal only } \\
\text { - Orientation: } \mathrm{n} / \mathrm{a} \text {. }\end{array}$ \\
\hline
\end{tabular}

entropy dependent on the angle with respect to galaxy orientation. We have focused on the TNG simulations because previous analyses had pointed towards an anisotropy of e.g. the galactic-wind mass outflow rates (Nelson et al. 2019b), CGM metallicities (Péroux et al. 2020), and of satellite galaxy quiescence (Martín-Navarro et al. 2021).

A similar outflow directionality, for example, has been found in the EAGLE simulation, with larger outflow rates occurring orthogonal to galactic disks (Mitchell et al. 2020). We therefore investigate how the TNG predictions compare with results from other cosmological simulations, specifically, from the original Illustris and EAGLE simulations (see Section 2.6). All three have non-negligibly different models for both stellar and SMBH feedback, and Table 1 gives a comparative overview.

In Figure 6 we contrast the outcome from Illustris (red), TNG (i.e. TNG100, blue), and EAGLE (green) in the anisotropy of gas column density (top), mass-weighted temperature (middle), and massweighted metallicity (bottom) across a wide range of halo mass $\left(M_{200 \mathrm{c}} \sim 10^{11.5}-10^{13.5} M_{\odot}\right)$. For this comparison we select simulated galaxies at $z=0$ and measure their median minor-to-major ratios at a radial distance $r \sim 0.5 R_{200 \mathrm{c}}$. We quantify and comment on the comparison at other galactocentric distances via Figure A2.

Illustris and EAGLE predict quite different mass dependences of the CGM density anisotropy compared to TNG, especially for galaxies with $M_{200 \mathrm{c}} \lesssim 10^{12.7} M_{\odot}$. On the one hand, the Illustris model predicts no substantial angular dependence across the whole mass range (with amplitude $\lesssim 0.05 \mathrm{dex}$ ). On the other hand, the CGM density anisotropy according to EAGLE - whereby the CGM is underdense along galaxies' minor axes, as in TNG - is most prominent at the lowest mass bin $M_{200 \mathrm{c}} \sim 10^{11.5} M_{\odot}$ and decreases toward higher masses.

Similarly, the quantitative predictions for the temperature angular modulation differ across the three models. Illustris predicts minorto-major $T_{\mathrm{mw}}$ ratios very close to unity; i.e. almost isotropic. The EAGLE model, like TNG, generally predicts hotter CGM orthogonal to galactic disks, but the anisotropy is strongest at lower masses than in TNG and the signal decreases with increasing mass.

In agreement with the findings of Péroux et al. 2020, EAGLE and TNG predict rather consistent CGM metallicity anisotropies, in both cases with monotonically-decreasing angular modulation with increasing mass. On the other hand, at the galactocentric distance of $r \sim 0.5 R_{200 \mathrm{c}}$, Illustris galaxies exhibit no significant anisotropy in the gas metallicity across the whole mass range ( $\lesssim 0.05 \mathrm{dex})$.

Overall, the Illustris model predicts no significant anisotropy in the CGM properties at galactocentric distances of $r \sim 0.5 R_{200 \mathrm{c}}$. However, interestingly, at smaller distances $\left(r \sim 0.25 R_{200 \mathrm{c}}\right)$, Illustris predicts strong angular modulations of both gas density and temperature ( $\sim 0.4$ dex, see Figure A2), particularly so for low-mass galaxies but in opposite senses compared to TNG or EAGLE. In Illustris, the CGM around low-mass galaxies is denser, colder, and metal richer along the minor axis than along the disks. We interpret these differences to be due to a combination of two aspects of the Illustris stellar feedback model which differ from TNG: i) the injection of cold winds, and ii) the bi-polar orientation of the winds at launch. The latter aspect had been discussed in the TNG methods paper (Pillepich et al. 2018a, see their Figure 5), but not fully quantified in terms of its possible effects on the CGM. It should be noted, however, that in general the Illustris feedback model is strongly disfavored based on comparisons of its outcome to galaxy demographics such as the galaxy color bimodality at the high-mass end, the galaxy stellar mass function at $z=0$, and the halo gas fraction within group-mass haloes (Vogelsberger et al. 2014a; Genel et al. 2014).

TNG and EAGLE qualitatively agree in terms of CGM geometry, with generally lower densities, higher temperatures, and higher metallicities along the minor axes of galaxies in both models. However, the two simulations return different mass dependences of the anisotropic signals. Whereas in TNG the CGM density and temperature anisotropies are maximal at intermediate, Milky Way-like masses of $M_{*} \sim 10^{10.5-11} M_{\odot}$ i.e. $M_{200 \mathrm{c}} \sim 10^{12.1-12.5} M_{\odot}$, EAGLE predicts monotonic mass trends for all the studied CGM properties. As already pointed out, these are the mass scales where observed galaxies transition from being mostly star-forming to being mostly quiescent and where the effects of SMBH feedback may start to be effective, in reality as well as in the models. Therefore, the differences in the CGM structure for galaxies with $M_{*} \gtrsim 10^{10.5} M_{\odot}$ $\left(M_{200 \mathrm{c}} \gtrsim 10^{12} M_{\odot}\right)$ in EAGLE and TNG are undoubtedly due to the different implementations of SMBH feedback.

Unlike in TNG, EAGLE injects SMBH feedback purely in the form of thermal energy, with non-continuous (i.e. bursty) events. SMBHs store up sufficient energy until they can change the temperature of nearby gas by a specified amount. It appears that as galaxies begin to quench in EAGLE this mechanism does not change in behavior or operation in any significant way (Bower et al. 2017), resulting in the monotonic mass trends described above. Importantly, the mass outflow rates of galactic winds in EAGLE massive galaxies are lower than those in TNG, at least at intermediate redshifts (Mitchell et al. 2020), pointing to the possibility that the SMBH thermal feedback 
in EAGLE is not as ejective as the SMBH kinetic feedback in TNG. Together, these effects explain how, at the transitional mass scale of $M_{200 \mathrm{c}} \sim 10^{12} M_{\odot}$, EAGLE can produce weaker angular modulations of the CGM density and temperature compared to TNG. Finally, we also note that at the low-mass end, stellar feedback appears to more strongly perturb CGM properties in EAGLE, in comparison to TNG.

Overall, we conclude that the explored CGM anisotropies may be a powerful diagnostic to discriminate among different feedback models in cosmological simulations, or at least among their effects: at the low-mass end, the CGM anistropic signals are sensitive to stellar feedback, whereas at the high-mass end, they can help distinguish among different SMBH feedback models.

\section{TESTS OF CGM ANISOTROPY VIA X-RAY OBSERVATIONS}

X-ray observations can probe the thermodynamical state of the gaseous atmospheres. In principle, from X-ray spectroscopic data it would be possible to derive the amount of hot gas, the gas temperature and metallicity. In that case, we could make comparison between simulations and observations on X-ray emission-weighted-like quantities, as provided for TNG100 at $z=0$ in Figure B1. Unfortunately, such spectroscopic data is currently not available for galaxies below the group mass scale $\left(M_{200 \mathrm{c}} \lesssim 10^{13} M_{\odot}\right)$. Therefore, in the following, we explore potential tests to (in)validate the predictions for the CGM anisotropy presented in this paper by focusing on X-ray emission data.

\subsection{CGM X-ray luminosity anisotropy}

In Figure 7, we quantify the predicted angular modulation of the intrinsic X-ray luminosity from the CGM gas in Illustris, TNG, and EAGLE. We show in the top row the minor-to-major ratios in X-ray surface brightness $\left(\Sigma_{\mathrm{X}}\right)$ as a function of halo mass at $z=0$, at the galactocentric distance $r \sim 0.5 R_{200 \mathrm{c}}$, while in the bottom row we show stacked X-ray surface brightness maps in [0.6-1.0] keV band at the mass bin $M_{20 c}=10^{12.3 \pm 0.1} M_{\odot}$ separately for TNG, EAGLE, and Illustris.

The resulting predictions are not trivial, as the X-ray luminosity of the halo gas depends to different degrees on gas density, temperature and metallicity, but, as shown in the previous Sections, the angular modulations of such gas physical properties may unfold in various and even opposing manners. We hence explore the anisotropy of $\Sigma_{\mathrm{X}}$ across different energy bands: from left to right, in the $[0.3-0.6]$ $\mathrm{keV},[0.6-1.0] \mathrm{keV},[1.0-2.3] \mathrm{keV}$, and [2.3 - 10.0] keV bands. These are energy ranges which the eROSITA telescope is sensitive to (Predehl et al. 2020), each of which is sensitive to the contributions from different gas phases.

For all the considered energy bands, the Illustris model predicts consistently no significant difference in $\Sigma_{X}$ measured along the minor vs. the major axes of galaxies, across the considered mass range.

On the other hand, for TNG and EAGLE, the X-ray emission from the CGM has non-negligible levels of angular modulation, particularly for low-mass systems $\left(M_{200 \mathrm{c}} \lesssim 10^{12.0-12.5} M_{\odot}\right)$. More intriguingly, the X-ray anisotropy, as well as its associated scatter, become progressively stronger toward harder energy bands, for both TNG and EAGLE but more so for the latter. In fact, at the transitional mass regime of $M_{*} \sim 10^{10.5-11} M_{\odot}$ or $M_{200 \mathrm{c}} \sim 10^{12.0-12.5} M_{\odot}$ and even in the soft bands (e.g. [0.6-1.0] keV), TNG predicts the X-ray surface brightness of the CGM located along the minor axis of galaxies to be about two times higher than that of gas located along the major axis (minor-to-major ratios of $\sim 0.25 \mathrm{dex}$ ). The corresponding anisotropy is instead weaker, if not vanishing, according to EAGLE $(\sim 0.20$ dex). Furthermore, it is interesting to notice that the anisotropy of the $\mathrm{X}$-ray emission does not necessarily reflect the anisotropy of the gas density; i.e. the former can be enhanced along the minor axis where the latter is lower.

The enhancement along the minor axis of the soft X-ray emission, e.g. in the $[0.3-0.6] \mathrm{keV}$ band, in low-mass TNG and EAGLE haloes, as well as the lack of anisotropy in Illustris, predominantly reflects the trends and angular modulations of the CGM metallicity. In $M_{200 \mathrm{c}} \sim 10^{11.5} M_{\odot}$ systems, the gas temperature is about $10^{5} \mathrm{~K}$, and most of the soft X-ray emission comes from metal lines. On the other hand, the $\mathrm{X}$-ray emission in harder bands is mainly driven by gas with high temperature, so that the anisotropy of the hard-band $\Sigma_{X}$ instead reflects the temperature modulation: see Figure 6, middle panel, vs. Figure 7, rightmost panel. The X-ray comparisons among Illustris, TNG100, and EAGLE at different galactocentric distances are given in Appendix A, Figure A3, and are qualitatively similar to the results of Figure 7.

\subsection{X-ray hardness}

The results above clearly suggest that another X-ray observable may be a key probe of the CGM anisotropy: the X-ray hardness. In Figure 8 we therefore show the angular modulation of the X-ray hardness, defined as the ratio of X-ray emission in the hard $[1.0-2.3]-\mathrm{keV}$ band to the soft band, $[0.3-0.6] \mathrm{keV}$.

The top panel quantifies the minor-to-major ratio of the X-ray hardness as a function of $M_{200 \mathrm{c}}$ at $r \sim 0.5 R_{200 \mathrm{c}}$. The main result is that the anisotropy of the X-ray hardness faithfully reflects the trends and angular modulations of the CGM temperature, except that the amplitude of the former is significantly larger $(\sim 0.5$ dex vs. $\sim 0.1 \mathrm{dex})$. The Illustris model predicts, as expected, generally no anisotropic hardness. On the other hand, the signals in TNG and EAGLE are somewhat distinct, with the hardness anisotropy in the former peaking at the usual transitional mass scale (with approximate amplitude of $\sim 0.5 \mathrm{dex})$ and that in EAGLE being most prominent toward the low-mass end $(\sim 0.6-0.8 \mathrm{dex})$. At high mass, the TNG signal is stronger than in EAGLE. The X-ray hardness anisotropy predicted by the different models at additional radii is shown in Figure A1.

Importantly, we note that the anisotropic signals in the X-ray hardness presented in Figure 8, as well as the signals in the X-ray surface brightness of Figure 7, are preserved, if not larger, when the minorto-major ratios are measured based on median stacked maps instead of individual galaxy maps (as described in Section 2.5). The former method is particularly relevant for the observational opportunities discussed in the next Section: as shown there, in practice it will not be possible to detect the anisotropic signal in X-ray at the individualgalaxy level, but only via stacking of a sufficiently-large number of galaxies.

To visually illustrate the different predictions from the three simulation models, the bottom panels of Figure 8 show stacked maps of the X-ray hardness for $M_{200 c}=10^{12.3 \pm 0.1} M_{\odot}$ haloes. We hence focus on the regime where SMBH feedback is at the origin of the differences among the models (see arguments in Sections 3.3 and 4). Visually, we clearly see that TNG predicts a larger angular modulation in X-ray hardness than EAGLE, with less central concentration, whereas there is no significant anisotropy in the Illustris map. 

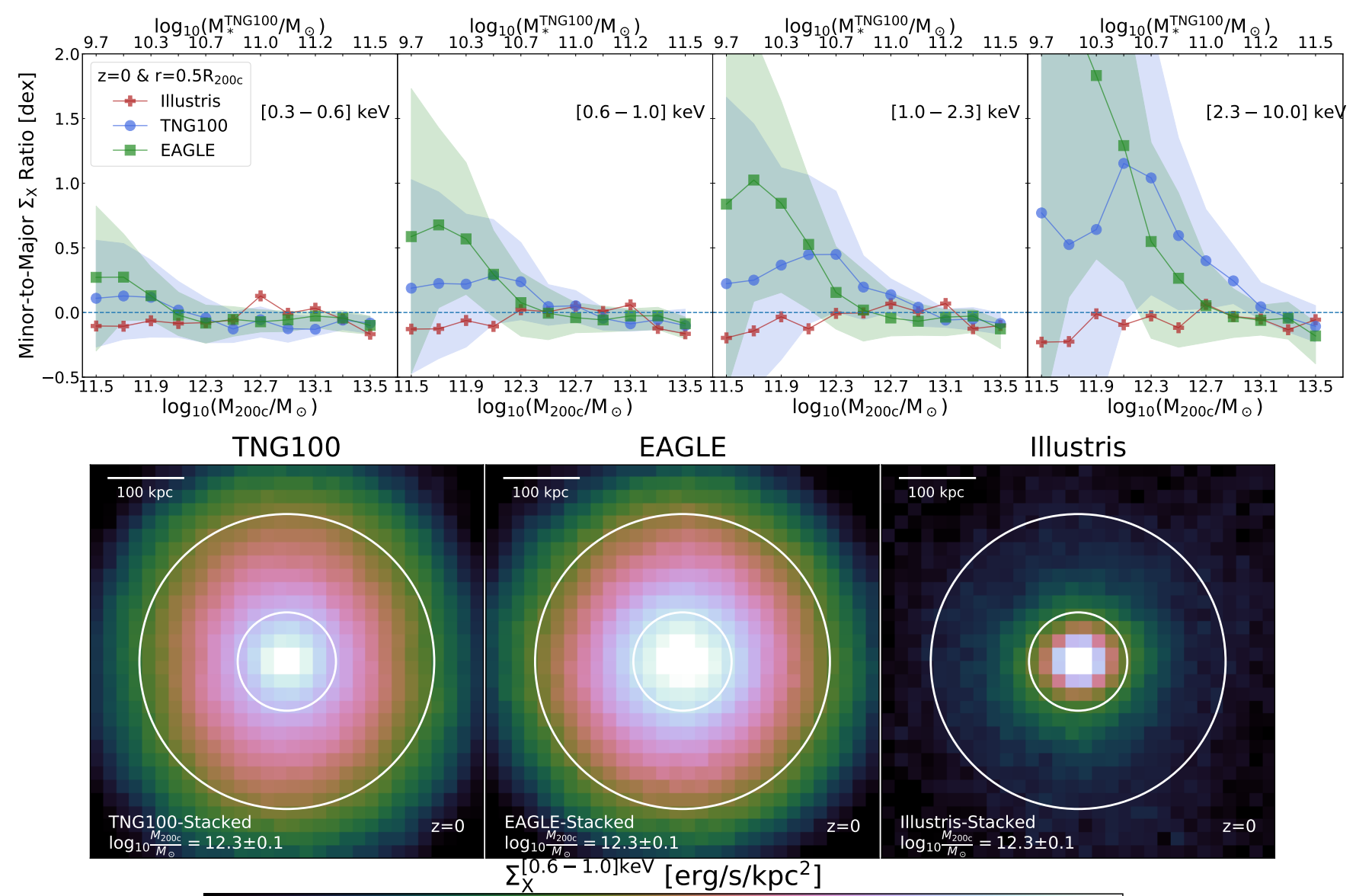

Illustris-Stacked
$\log _{10} \frac{M_{200 c}}{M}=12.3 \pm 0.1$

30.0

31.5

33.0

34.5

Figure 7. CGM anisotropy in X-ray surface brightness, for Illustris, TNG, and EAGLE simulated galaxies at $z=0$. Top: From left to right we show the minor-to-major ratio of the X-ray surface brightness $\left(\Sigma_{X}\right)$ in four different energy bands: $[0.3-0.6] \mathrm{keV},[0.6-1.0] \mathrm{keV},[1.0-2.3] \mathrm{keV}$, and [2.3 - 10.0] $\mathrm{keV}$, respectively. Annotations are as in Figure 6. The top x-axes represent median values of stellar mass for TNG galaxies (note the scale is not linear). Bottom: Stacked edge-on maps of the X-ray surface brightness in $[0.6-1.0] \mathrm{keV}$ for galaxies with $\boldsymbol{M}_{200 \mathrm{c}}=10^{12.3 \pm 0.1} \boldsymbol{M}_{\odot}$. From left to right we show: TNG100, EAGLE and Illustris. The two circles specify galactocentric distances at $0.25 R_{200 \mathrm{c}}$ and $0.75 R_{200 \mathrm{c}}$. In the soft bands, e.g. [0.6 - 1.0] keV (second panel from the left in the top row), the CGM of massive galaxies $\left(M_{200 \mathrm{c}} \simeq 10^{12.1-12.5} M_{\odot}\right)$ is X-ray brighter in directions orthogonal to the stellar "disks", by about a factor of two. For both TNG and EAGLE, harder bands reveal stronger angular modulations of X-ray luminosity.

\subsection{X-ray observational opportunities}

The predictions from the three simulation models studied in this paper are most discrepant either at the low-mass end $\left(M_{200 c} \sim 10^{11.5} M_{\odot}\right)$ or at the transitional mass range $\left(M_{200 \mathrm{c}} \sim 10^{12.0-12.5} M_{\odot}\right)$, where the TNG anisotropic signals peak. In the following, we show that the latter is within the reach of upcoming X-ray observations.

We consider a number of stacking experiments to examine the observability of the predicted X-ray signals via the eROSITA all-sky survey. We are interested in signals from the CGM region bracketed by $[0.25-0.75] \mathrm{R}_{200 \mathrm{c}}$, which is most relevant to the anisotropic signals presented in this paper. In addition, to study the azimuthal dependence of the signals, we aim to characterize CGM areas spanning at most $\sim 1 / 12$ of the $2 \pi$ circumference, in order to constrain how the X-ray emission varies between the major and minor axes for three different azimuthal angles.

In Figure 9, we show the eROSITA mock X-ray count rates in the $[0.5-2.0] \mathrm{keV}$ band for TNG galaxies, as a function of halo mass and galaxy stellar mass (top axis). The median count rates are obtained based on TNG100 galaxies at each of the three indicated redshifts following the method described in Section 2.2. To assess the detectability we also derive Poisson noise (error bars) and the signal-to-noise ratio for each mass bin (denoted by filled and empty cicles) based on the source and background photon counts. The latter is calculated assuming an X-ray eROSITA background level of $2.1 \times 10^{-3}$ counts $/ \mathrm{s} / \operatorname{arcmin}^{2}$ in the energy band of $[0.5-2.0] \mathrm{keV}$ (Merloni et al. 2012). The photon counts are obtained by stacking a certain number of galaxies in each mass bin, corresponding to the total number of galaxies available up to the given redshift (specified at the extra axes on the top of the plot $^{2}$ ) and assuming $2 \mathrm{ks}$ observing time. In addition, the Poisson noise (error bars) and signal-to-noise ratio are computed for the intentionally-detected area; i.e. 1/12 of the $[0.25-0.75] R_{200 c}$ annulus, accounting for the fact that we treat the 4 quadrants identically.

According to Figure 9, if we stack 10000 galaxies out to redshift

2 The number of available galaxies within a certain distance is calculated based on the TNG100 mass function, corrected by the relevant volume factor. We also exclude face-on galaxies ( $\sim 1 / 3$ of the total number of galaxies), which are not useful for detecting anisotropic signals. 


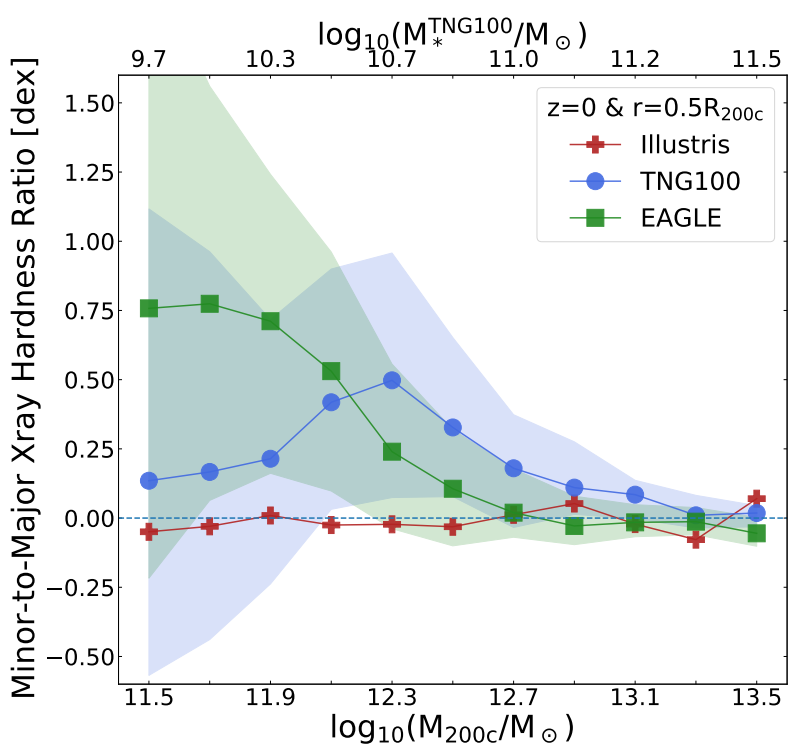

TNG100

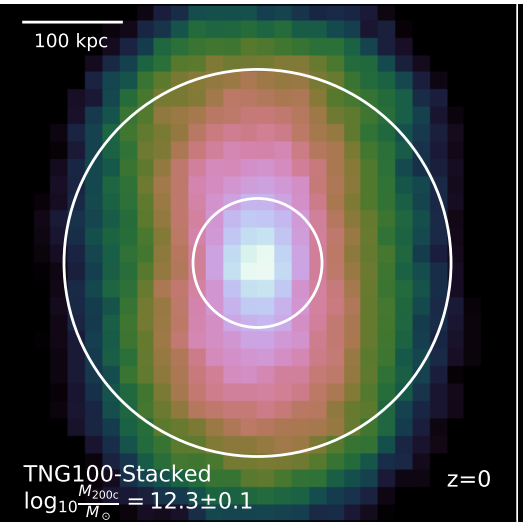

EAGLE

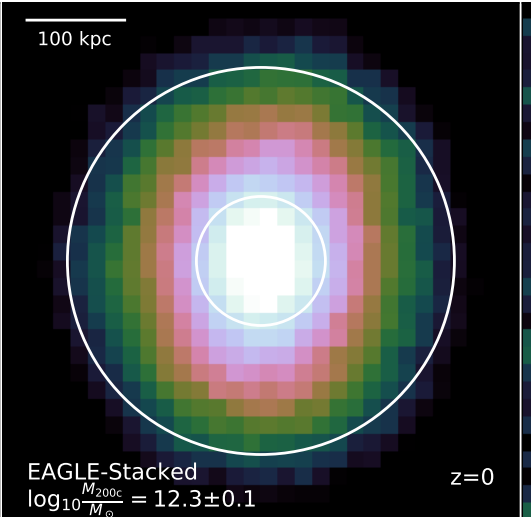

Illustris

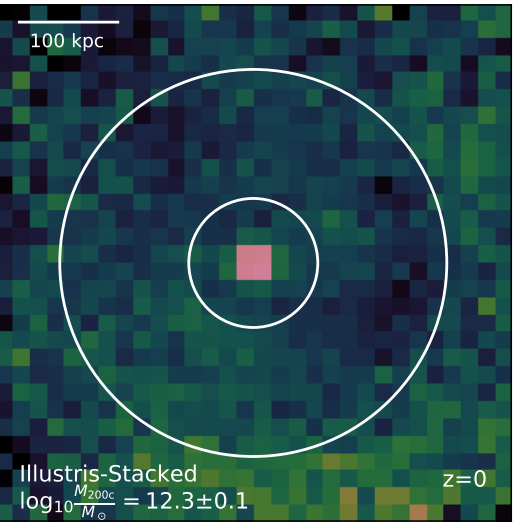

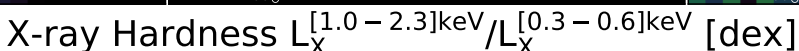

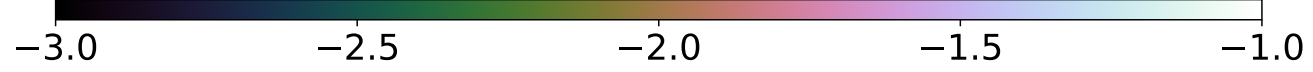

Figure 8. CGM anisotropy in X-ray hardness, for Illustris, TNG, and EAGLE simulated galaxies at $z=0$. Top: The minor-to-major axes ratio of X-ray hardness as a function of galaxy/halo mass. The hardness is defined as the ratio of the X-ray emission in the hard band ([1.0 - 2.3] keV) to the soft band ([0.3 - 0.6] $\mathrm{keV})$. Annotations are as in Figure 6. For clarity, shaded areas, i.e. the scatter, are given only for TNG and EAGLE. In addition, the top x-axes show median values of stellar mass for TNG galaxies (note the scale is not linear). Bottom: Stacked edge-on maps of the X-ray hardness in galaxies with $M_{200 c} \sim 10^{12.3} M_{\odot}$, for TNG100, EAGLE and Illustris separately. The two concentric circles specify galactocentric distances of $0.25 R_{200 \mathrm{c}}$ and $0.75 R_{200 \mathrm{c}}$. The anisotropy of the $\mathrm{X}$-ray hardness in the CGM is a promising observable to discriminate among different SMBH feedback models.

$z=0.1$, then TNG predicts a detectable X-ray signal down to the mass range of $M_{200 \mathrm{c}} \sim 10^{12.3} M_{\odot}\left(M_{*} \sim 10^{10.7} M_{\odot}\right)$ with SNR $\gtrsim 3$ (orange), corresponding to roughly 400 source photons detected per $\operatorname{arcmin}^{2}$. This number of galaxies is within the reach of current galaxies survey, e.g. the Sloan Digital Sky Survey (SDSS, Alam et al. 2015; Saulder et al. 2016), while larger future spectroscopic galaxy surveys will increase the sample statistics further. Based on our findings summarized in Figure 9, the upcoming eROSITA allsky survey therefore has the potential to verify the X-ray anisotropy in the CGM predicted by state-of-the-art cosmological simulations for intermediate- and high-mass galaxies $\left(M_{200 \mathrm{c}} \gtrsim 10^{12.0-12.5} M_{\odot}\right)$, directly discriminating among different models of SMBH feedback. On the other hand, despite the improved spatial resolution, stacking on nearby galaxies out to $40 \mathrm{Mpc}$ (green) or $100 \mathrm{Mpc}$ (blue) with only $2 \mathrm{ks}$ of exposure would result in a detectable signal achievable only for significantly more massive halos, due to the decreased statistics.
Finally, observations for the low-mass end $\left(M_{200 c} \sim 10^{11.5} M_{\odot}\right.$ or $\left.M_{*} \sim 10{ }^{10} M_{\odot}\right)$ will be significantly more challenging. Galaxy samples larger than those of Figure 9 are needed to ensure detection via stacking: this in turn will require extending the observations to farther distances $(z>0.1)$. Unfortunately, at such distances, eROSITA will not be able to spatially resolve their anistropic signal. Observations of low-mass systems will be perhaps possible only with next-generation X-ray observatories (e.g. Simionescu et al. 2021). This regime probes distinct anisotropy signals, as predicted by the three simulations considered in this paper, and would further constrain the impact of stellar feedback models on the circumgalactic medium of galaxies. 


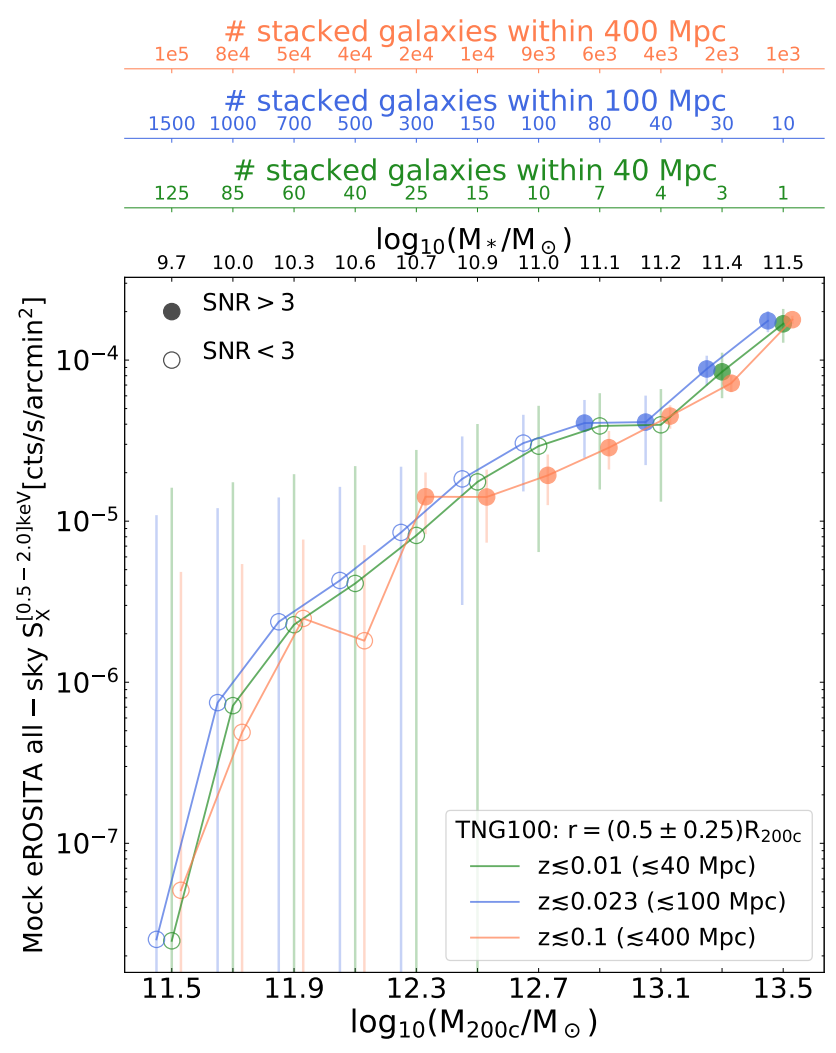

Figure 9. Mock eROSITA survey count rates as a function of halo mass for TNG100 galaxies, based on different assumed surveys/redshifts (three colors). The circles represent the median count rate at each mass bin, and the error bars denote $\sim 1 \sigma$ Poisson noise based on both source and background photon counts. Filled (empty) circles specify signal-to-noise ratio (SNR) above (below) the value of 3 . The values of SNR and Poisson noise are computed for the intentionally detected area (see text) and based on the photon counts obtained from stacking the number of galaxies available within a certain distance (colored top axes), assuming a $2 \mathrm{ks}$ observing time per galaxy.

\section{SUMMARY AND CONCLUSIONS}

In this paper we have used the IllustrisTNG simulations to provide theoretical, quantitative predictions as to how feedback from the central regions of galaxies affects the thermodynamical and chemical properties of surrounding gas - the circumgalactic medium (CGM). In particular, we study how CGM properties - density, temperature, metallicity, as well as pressure, entropy, and X-ray emission - depend on azimuthal angle, defined as the galactocentric angle with respect to the central galaxy's stellar minor axis.

We have studied galaxies across the galaxy stellar mass range of $10^{10-11.5} M_{\odot}$ and thus focused on the high-mass regime $(\gtrsim$ $10^{10.5} M_{\odot}$ ) where SMBH feedback is expected to be the dominant mechanism that drives galactic outflows and where testing opportunities via X-ray observations are feasible. We have quantified the CGM anisotropy at $z=0$ via stacking and through population averages, as a function of galactocentric distance, across a wide range of galaxy and halo masses, and have compared the TNG predictions to those from two other simulations, Illustris and EAGLE.

Our main findings are as follows:

- The TNG model predicts that the CGM of massive galaxies at the transitional mass regime of $M_{*} \simeq 10^{10.5}-10^{11.0} M_{\odot}$ is markedly anisotropic (Figures 1 and 4), with gas along the minor axis of galaxies being more diluted, hotter and more metal rich than the gas along galactic planes. The amplitude of this angular modulation is non-negligible $(0.1-0.3 \mathrm{dex})$, is clearly seen by stacking across large number of galaxies, and persists out to the virial radius of haloes ( $260 \mathrm{kpc}$ on average). Our work suggests that the Milky Way's mass range is the sweet spot where the CGM anisotropy is most prominent because here two fundamental physical conditions occur: i) The presence of sufficiently-strong, feedback-driven outflows, as those produced in the TNG model by the kinetic SMBH feedback; ii) the presence of a gaseous disk in the inner regions of galaxies that can (re)direct outflows in bi-polar directions.

- For $M_{*} \simeq 10^{10.5} M_{\odot}$ TNG galaxies, the CGM anisotropy in gas density and temperature is remarkably more pronounced for galaxies that are quenched, host an overmassive SMBH with respect to the average, or host a SMBH that has injected a larger-than-average amount of energy in the kinetic feedback mode, which in turn produces strong SMBH-driven winds (Figure 4). This supports the picture whereby, in TNG, SMBH-driven outflows are the physical driver of the angular modulation of CGM density and temperature at the high-mass end. In fact, the strength of the gas-metallicity anisotropy exhibits opposite correlations with those galaxy properties that are a measure of SMBH activity.

- According to the TNG model, the CGM is indeed anistropic in its thermodynamical properties and chemical content over a large galaxy mass range, $M_{*} \simeq 10^{10}-10^{11.5} M_{\odot}$ (Figure 3), and it is generally more pronounced at smaller galactocentric distances. In TNG the metallicity angular modulation is stronger for lower-mass galaxies, and overall decreases with increasing galaxy mass. In contrast, the density and temperature anisotropies are maximal at the aforementioned transitional mass scale; i.e. at or slightly above the Milky Way-mass scale, corresponding to halo masses of $M_{200 c} \sim 10^{12.0-12.5} M_{\odot}$ and SMBH masses of $M_{\mathrm{BH}} \sim 10^{8.0-8.5} M_{\odot}$. We speculate that the CGM is more isotropic at both higher and lower masses because of the less effective stellar and SMBH-driven thermal feedback at the low-mass end and of the relative isotropy of the inner gas distribution at the high-mass end.

- In general the CGM is more diluted along the minor axis, but its temperature is enhanced, making the halo gas pressure essentially isotropic. On the other hand, the CGM entropy maximally captures, via its strong angular modulation, the imprints of feedback (Figure 5).

- In comparison to TNG, the original Illustris simulation returns negligible, if not, vanishing levels of angular dependence of the CGM properties, throughout the studied mass range (Figure 6).

- On the other hand, the EAGLE model, despite the very different implementation of both stellar and SMBH feedback, shows many qualitatively-similar results: gas density is generally lower, and temperature and metallicity higher, orthogonal to galaxy disks. However, quantitatively, EAGLE and TNG predict different levels of CGM anisotropy and very different mass trends (Figure 6). EAGLE produces more prominent anisotropies in the CGM density and temperature at the low-mass end, but also weaker, if not vanishing, CGM angular modulations for massive galaxies, $M_{200 c} \gtrsim 10^{12} M_{\odot}$.

Our paper demonstrates that the $z=0$ angular modulation of the CGM properties is highly sensitive to the way energy from stellar and $\mathrm{SMBH}$ feedback is injected into the surrounding gas. It thus provides a promising test-bed to discriminate among galaxy feedback models 
and to (in)validate the outcome of current and future state-of-theart cosmological galaxy simulations. This is particularly the case for massive galaxies and hence for SMBH feedback. For $M_{200 \mathrm{c}} \gtrsim$ $10^{12.3} M_{\odot}$ or $M_{*} \gtrsim 10^{10.7} M_{\odot}$, the eROSITA telescope, via its allsky survey, should be able to detect X-ray emission from the CGM via stacking, across the relevant halo scales (Figure 9). In particular, in terms of X-ray observational signatures, we find that:

- The anisotropy of CGM density, temperature and metallicity imprints complex and informative signatures in the angular modulation of the X-ray surface brightness of the simulated galaxies (Figure 7). Whereas Illustris predicts isotropy, the CGM of TNG (EAGLE) $M_{*} \simeq 10^{10.5-11} M_{\odot}$ galaxies is $\sim 1.8$ (1.6) times brighter along the minor versus major axis for the $[0.6-1.0] \mathrm{keV}$ band, with angular modulation increasing towards harder bands.

- The X-ray hardness of the CGM, defined as the ratio of X-ray emission between hard (e.g. [1.0 - 2.3] keV) and soft (e.g. [0.3$0.6] \mathrm{keV}$ ) bands, is a promising observable to discriminate among different SMBH feedback models (Figure 8), exhibiting a marked angular dependence of $\sim 0.5 \mathrm{dex}$ at the transitional mass scale.

Within the interpretative framework of IllustrisTNG, the CGM anisotropy that we have quantified in this paper - if in place also in the Universe - would be the manifestation across the galaxy population of the bipolar bubbles that eROSITA has detected in Xray emission above and below the disk of our Galaxy (Predehl et al. 2020), which themselves trace the Fermi bubbles (Su et al. 2010). In fact, the TNG model predicts coherent, dome-like features of overpressurized gas that impinge into the CGM above and below the disks of most Milky Way- and Andromeda- like galaxies (Pillepich et al. 2021) of the TNG50 simulation (Pillepich et al. 2019; Nelson et al. $2019 b$ ), whereby the gas within the bubbles is under dense, $10^{6-7} \mathrm{~K}$ hot and enriched to metallicities of $0.5-2 Z_{\odot}$.

The detection of X-ray anisotropy in the CGM of Milky Way-mass galaxies in the Universe would constitute an indirect confirmation of the predicted ubiquity of eROSITA-like bubble features in external individual galaxies (Pillepich et al. 2021), and place stringent constraints on the current implementation, or at least manifestations, of SMBH feedback in simulations.

In fact, the novel phenomenon uncovered in SDSS data by MartínNavarro et al. 2021, dubbed "anisotropic satellite galaxy quenching", and in place also in the TNG but not in the original Illustris simulations, already provides compelling support to the ejective character of SMBH feedback. The lack of anisotropy in the CGM density of Illustris galaxies (Figure 6, top panel, and Figure A2) further supports the interpretation that the anisotropic satellite-quenching signal is driven by the clearing of halo gas preferentially in the direction of the minor axis of central galaxies in groups because of the activity of their SMBHs.

\section{DATA AVAILABILITY}

The IllustrisTNG simulations are publicly available and accessible at www.tng-project.org/data (Nelson et al. 2019a). Similarly, data from the Illustris and EAGLE projects are available at www.illustris-project. org/data (Nelson et al. 2015) and e.g. http://eagle.strw.leidenuniv.nl/ (McAlpine et al. 2016), respectively. Data directly related to this publication and its figures is available on request from the corresponding author.

\section{ACKNOWLEDGEMENTS}

AP and NT thank Elad Zinger for useful conversations. DN acknowledges funding from the Deutsche Forschungsgemeinschaft (DFG) through an Emmy Noether Research Group (grant number NE 2441/1-1) and NT and AP acknowledge funding by the Deutsche Forschungsgemeinschaft (DFG, German Research Foundation) Project-ID 138713538 - SFB 881 ("The Milky Way System", subproject A01). NW is supported by the GACR grant $13491 \mathrm{X}$. The primary TNG simulations were carried out with compute time granted by the Gauss Centre for Supercomputing (GCS) under Large-Scale Projects GCS-ILLU and GCS-DWAR on the GCS share of the supercomputer Hazel Hen at the High Performance Computing Center Stuttgart (HLRS). Additional simulations and analyses had been carried out on the Isaac machine of the Max Planck Institute for Astronomy (MPIA) and on the other systems at the Max Planck Computing and Data Facility (MPCDF).

\section{REFERENCES}

Alam S., et al., 2015, ApJS, 219, 12

Barnes D. J., et al., 2018, MNRAS, 481, 1809

Booth C. M., Schaye J., 2009, MNRAS, 398, 53

Bower R. G., Schaye J., Frenk C. S., Theuns T., Schaller M., Crain R. A., McAlpine S., 2017, MNRAS, 465, 32

Byrohl C., Nelson D., Behrens C., Pillepich A., Hernquist L., Marinacci F., Vogelsberger M., 2020, arXiv e-prints, p. arXiv:2009.07283

Cattaneo A., et al., 2009, Nature, 460, 213

Crain R. A., et al., 2015, MNRAS, 450, 1937

Croton D. J., et al., 2006, MNRAS, 365, 11

Davé R., Anglés-Alcázar D., Narayanan D., Li Q., Rafieferantsoa M. H., Appleby S., 2019, MNRAS, 486, 2827

Davies J. J., Crain R. A., Oppenheimer B. D., Schaye J., 2020, MNRAS, 491, 4462

Di Matteo T., Springel V., Hernquist L., 2005, Nature, 433, 604

Donnari M., Pillepich A., Nelson D., Marinacci F., Vogelsberger M., Hernquist L., 2020, arXiv e-prints, p. arXiv:2008.00004

Eckert D., Gaspari M., Gastaldello F., Le Brun A. M. C., O'Sullivan E., 2021, Universe, 7, 142

Fabian A. C., 2012, ARA\&A, 50, 455

Genel S., et al., 2014, MNRAS, 445, 175

Harrison C. M., 2017, Nature Astronomy, 1, 0165

Henden N. A., Puchwein E., Shen S., Sijacki D., 2018, MNRAS, 479, 5385

Hlavacek-Larrondo J., et al., 2015, ApJ, 805, 35

Khalatyan A., Cattaneo A., Schramm M., Gottlöber S., Steinmetz M., Wisotzki L., 2008, MNRAS, 387, 13

Kirkpatrick C. C., Gitti M., Cavagnolo K. W., McNamara B. R., David L. P., Nulsen P. E. J., Wise M. W., 2009, ApJ, 707, L69

Kirkpatrick C. C., McNamara B. R., Cavagnolo K. W., 2011, ApJ, 731, L23

Kormendy J., Ho L. C., 2013, ARA\&A, 51, 511

Le Brun A. M. C., McCarthy I. G., Schaye J., Ponman T. J., 2014, MNRAS, 441,1270

Man A., Belli S., 2018, Nature Astronomy, 2, 695

Marinacci F., et al., 2018, MNRAS, 480, 5113

Martín-Navarro I., Pillepich A., Nelson D., Rodriguez-Gomez V., Donnari M., Hernquist L., Springel V., 2021, Nature, 594, 187

McAlpine S., et al., 2016, Astronomy and Computing, 15, 72

McCarthy I. G., et al., 2010, MNRAS, 406, 822

McNamara B. R., Nulsen P. E. J., 2012, New Journal of Physics, 14, 055023

Merloni A., et al., 2012, arXiv e-prints, p. arXiv:1209.3114

Mitchell P. D., Schaye J., Bower R. G., Crain R. A., 2020, MNRAS, 494, 3971

Morganti R., 2017, Frontiers in Astronomy and Space Sciences, 4, 42

Naab T., Ostriker J. P., 2017, ARA\&A, 55, 59

Naiman J. P., et al., 2018, MNRAS, 477, 1206

Nelson D., et al., 2015, Astronomy and Computing, 13, 12 
Nelson D., et al., 2018a, MNRAS, 475, 624

Nelson D., et al., 2018b, MNRAS, 477, 450

Nelson D., et al., 2019a, Computational Astrophysics and Cosmology, 6, 2

Nelson D., et al., 2019b, MNRAS, 490, 3234

Nelson D., et al., 2020, MNRAS, 498, 2391

Nelson E. J., et al., 2021a, arXiv e-prints, p. arXiv:2101.12212

Nelson D., Byrohl C., Peroux C., Rubin K. H. R., Burchett J. N., 2021b, arXiv e-prints, p. arXiv:2106.09023

Nulsen P., Jones C., Forman W., Churazov E., McNamara B., David L., Murray S., 2009, in Heinz S., Wilcots E., eds, American Institute of Physics Conference Series Vol. 1201, American Institute of Physics Conference Series. pp 198-201 (arXiv: 0909. 1809), doi:10.1063/1.3293033

O’Sullivan E., Giacintucci S., David L. P., Vrtilek J. M., Raychaudhury S., 2011, MNRAS, 411, 1833

Oppenheimer B. D., et al., 2020, ApJ, 893, L24

Péroux C., Nelson D., van de Voort F., Pillepich A., Marinacci F., Vogelsberger M., Hernquist L., 2020, MNRAS, 499, 2462

Pillepich A., et al., 2018a, MNRAS, 473, 4077

Pillepich A., et al., 2018b, MNRAS, 475, 648

Pillepich A., et al., 2019, MNRAS, 490, 3196

Pillepich A., Nelson D., Truong N., Weinberger R., Martin-Navarro I., Springel V., Faber S. M., Hernquist L., 2021, arXiv e-prints, p. arXiv:2105.08062

Planck Collaboration et al., 2016, A\&A, 594, A13

Predehl P., et al., 2020, Nature, 588, 227

Randall S. W., et al., 2011, ApJ, 726, 86

Reeves J. N., et al., 2009, ApJ, 701, 493

Saulder C., van Kampen E., Chilingarian I. V., Mieske S., Zeilinger W. W., 2016, A\&A, 596, A14

Schaye J., et al., 2015, MNRAS, 446, 521

Sijacki D., Vogelsberger M., Genel S., Springel V., Torrey P., Snyder G. F., Nelson D., Hernquist L., 2015, MNRAS, 452, 575

Silk J., Rees M. J., 1998, A\&A, 331, L1

Simionescu A., Werner N., Finoguenov A., Böhringer H., Brüggen M., 2008, A\&A, 482, 97

Simionescu A., Werner N., Böhringer H., Kaastra J. S., Finoguenov A., Brüggen M., Nulsen P. E. J., 2009, A\&A, 493, 409

Simionescu A., et al., 2021, Experimental Astronomy,

Smith R. K., Brickhouse N. S., Liedahl D. A., Raymond J. C., 2001, ApJ, 556, L91

Springel V., 2005, MNRAS, 364, 1105

Springel V., 2010, MNRAS, 401, 791

Springel V., Di Matteo T., Hernquist L., 2005, MNRAS, 361, 776

Springel V., et al., 2018, MNRAS, 475, 676

Su M., Slatyer T. R., Finkbeiner D. P., 2010, ApJ, 724, 1044

Terrazas B. A., et al., 2020, MNRAS, 493, 1888

Tombesi F., Cappi M., Reeves J. N., Palumbo G. G. C., Braito V., Dadina M., 2011, ApJ, 742, 44

Tombesi F., Cappi M., Reeves J. N., Braito V., 2012, MNRAS, 422, L1

Torrey P., et al., 2019, MNRAS, 484, 5587

Truong N., et al., 2018, MNRAS, 474, 4089

Truong N., et al., 2020, MNRAS, 494, 549

Truong N., Pillepich A., Werner N., 2021, MNRAS, 501, 2210

Tumlinson J., Peeples M. S., Werk J. K., 2017, ARA\&A, 55, 389

Vogelsberger M., Genel S., Sijacki D., Torrey P., Springel V., Hernquist L., 2013, MNRAS, 436, 3031

Vogelsberger M., et al., 2014a, MNRAS, 444, 1518

Vogelsberger M., et al., 2014b, Nature, 509, 177

Weinberger R., et al., 2017, MNRAS, 465, 3291

Weinberger R., et al., 2018, MNRAS, 479, 4056

Werner N., McNamara B. R., Churazov E., Scannapieco E., 2019, Space Sci. Rev., 215, 5

Zhang D., 2018, Galaxies, 6, 114

Zhuravleva I., et al., 2014, Nature, 515, 85

Zinger E., et al., 2020, MNRAS, 499, 768

van den Bosch R. C. E., 2016, ApJ, 831, 134
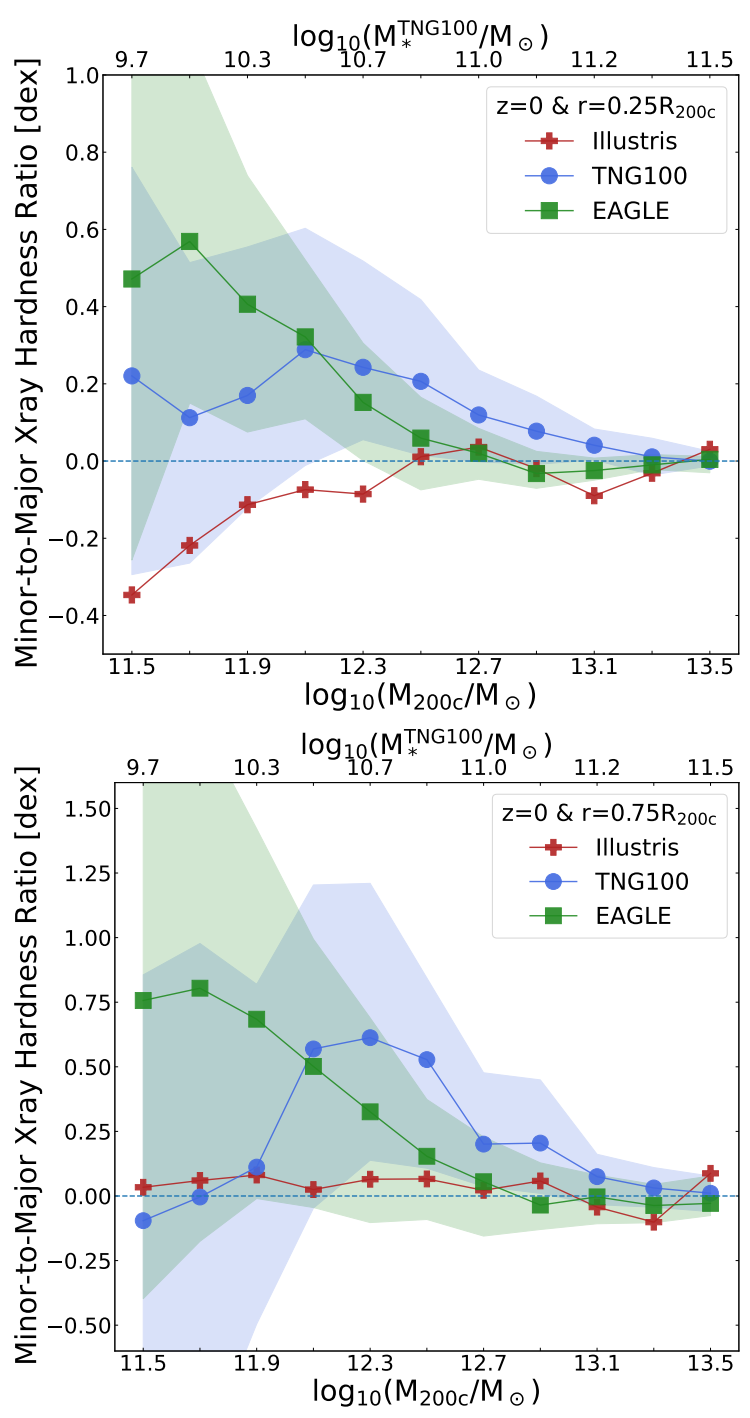

Figure A1. Similar to Figure 8 but for the measurements at other distances: $r=0.25 R_{200 \mathrm{c}}$ (top) and $r=0.75 R_{200 \mathrm{c}}$ (bottom).

\section{APPENDIX A: MEASUREMENTS AT DIFFERENT GALACTOCENTRIC DISTANCES}

In this Section we provide additional comparisons between TNG, EAGLE, and Illustris for measurements at other galactocentric distances of $0.25 R_{200 \mathrm{c}}$ and $0.75 R_{200 \mathrm{c}}$ : X-ray hardness (Figure A1), thermodynamics and metallicity content (Figure A2), and X-ray surface brightness (Figure A3).

Qualitatively, the comparison among the three simulations at large distance $r \sim 0.75 R_{200 c}$ is consistent with the results presented in the paper at $\mathrm{r} \sim 0.5 \mathrm{R}_{200 \mathrm{c}}$. On the other hand, at smaller radii $r \sim 0.25 R_{200 c}$, the Illustris simulation predicts anisotropic signals with significantly larger amplitudes at the low-mass end $\left(M_{200 \mathrm{c}} \lesssim 10^{12.3} M_{\odot}\right)$ compared to the results at the other two radii, where Illustris produces almost isotropic distribution of thermodynamic properties as well as metal content. For instance, concerning the gas temperature (top middle panel of Figure A2), the Illustris value of minor-to-major ratio at $M_{200 \mathrm{c}} \sim 10^{11.5} M_{\odot}$ is about -0.4 dex, compared to $\sim 0.15$ dex value of TNG and EAGLE, namely Illustris predicts that at small radii the gas is colder along the minor axis in contrast with TNG and EAGLE predictions. The opposite 
predictions of Illustris, in comparison to the other two simulations, could be explained by its stellar feedback model, in which cold gas is deposited preferably along the minor axis (see also discussion in Section 4).

\section{APPENDIX B: X-RAY EMISSION-WEIGHTED QUANTITIES}

We examine the anisotropic signals in gas temperature and metallicity when their averaged values are taken as X-ray emission-weighted mean. The mass dependence of those signals, which is similar to that shown in Figure 3 (middle column), are shown in Figure B1. The $\mathrm{X}$-ray emission is computed in the energy band [0.5 - 2.0] $\mathrm{keV}$.

In comparison to the mass-weighted quantities, the anisotropic signals in X-ray emission-weighted gas temperature and metallicity exhibit similar patterns. However, it is worth noting that the amplitude of emission-weighted temperature (metallicity) is overall larger by about $0.03(0.1)$ dex except for temperature signal measured at small radii $\left(r \sim 0.25 R_{200 c}\right)$. 

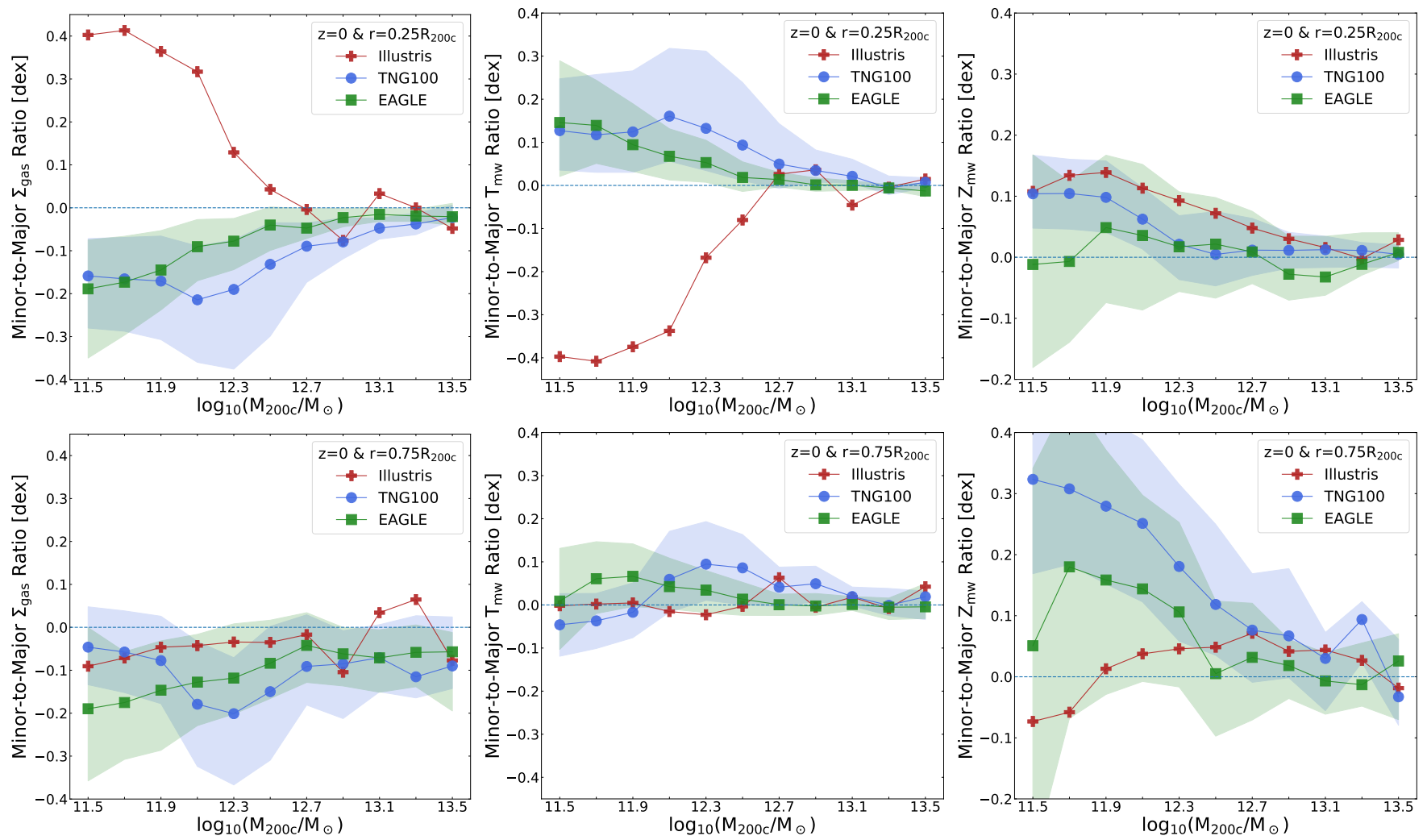

Figure A2. Similar to Figure 6 but for the measurements at different galactocentric distances: $r=0.25 R_{200 \mathrm{c}}$ (top row) and $r=0.75 R_{200 \mathrm{c}}($ bottom row).
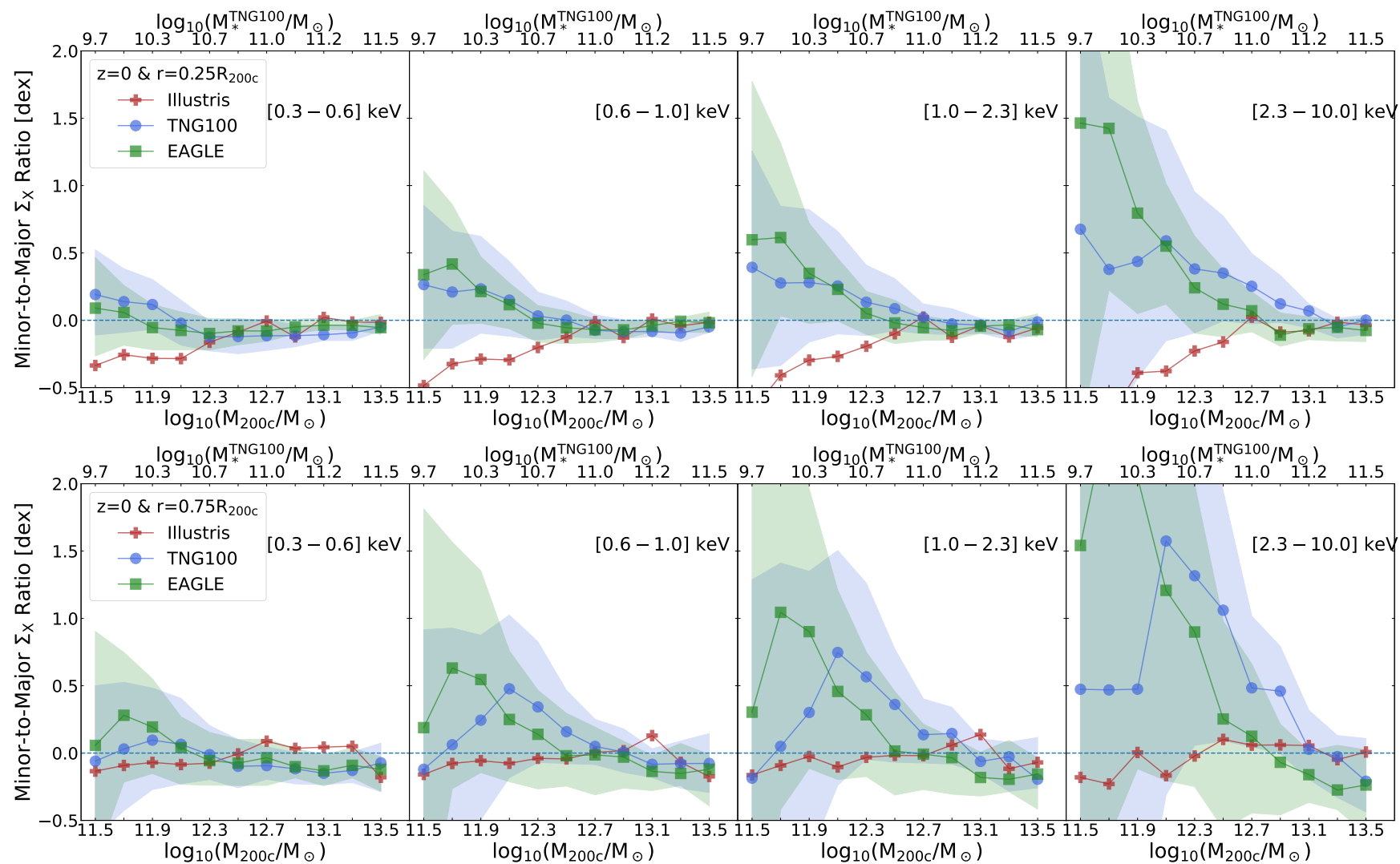

Figure A3. Similar to Figure 7 but for the measurements at other distances: $r=0.25 R_{200 \mathrm{c}}$ (top) and $r=0.75 R_{200 \mathrm{c}}($ bottom). 

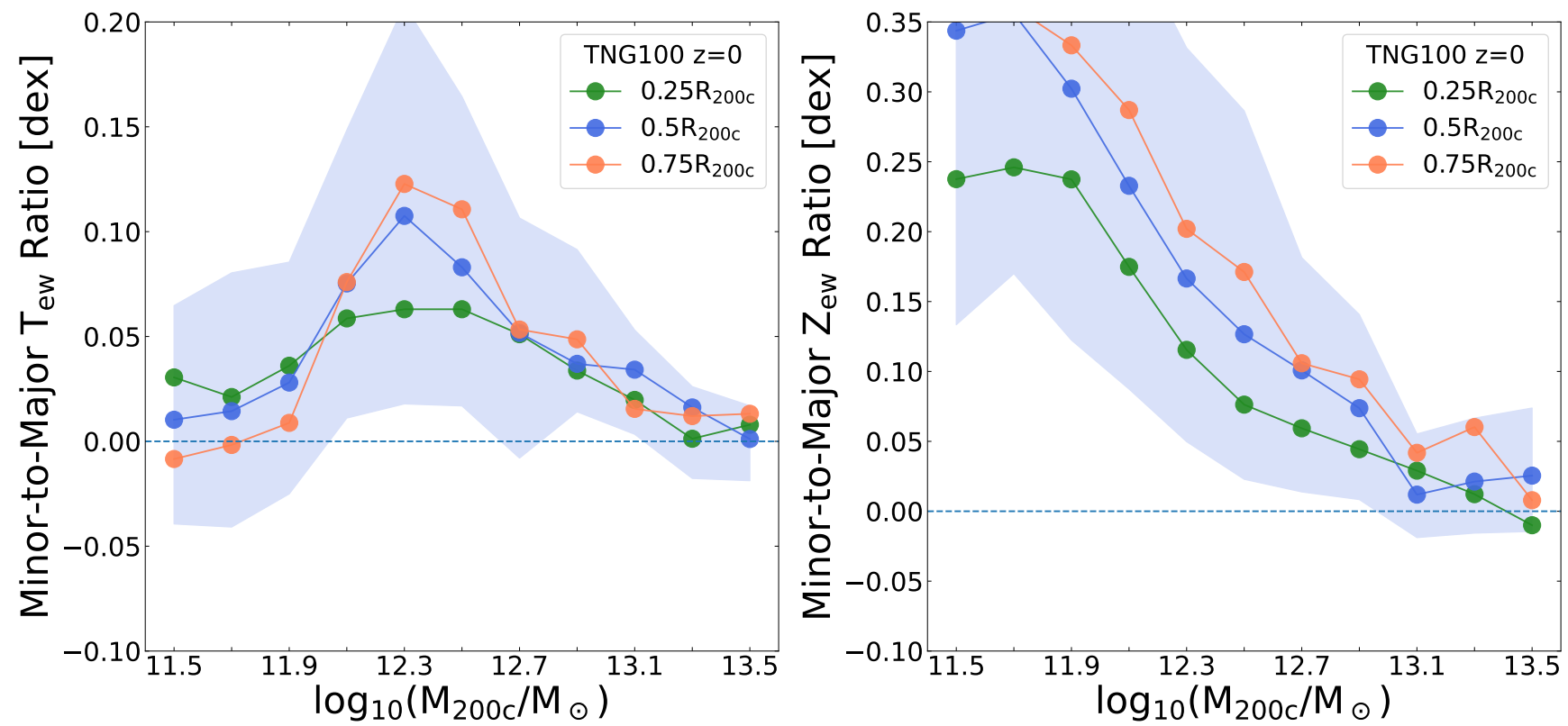

Figure B1. Mass dependence of the anisotropic signals in gas temperature (left) and metallicity (right) according to the TNG100 simulation. This figure is similar to Figure 3 (middle column), but gives results for CGM quantities that are averaged with weights given by the X-ray emissivity of the gas, in the [0.5 - 2.0] keV band. 